\title{
Update on prevention of mother-to-child HIV transmission
}

\author{
Lynne M. Mofenson \\ Pediatric, Adolescent and Maternal AIDS Branch, National Institute of Child Health and Human \\ Development, 6100 Executive Boulevard, Rockville, MD
}

The pediatric HIV epidemic in the US and other more developed countries changed dramatically after February 1994, when the results of PACTG 076 demonstrated that a triple regimen of ZDV reduced the risk of perinatal transmission by nearly $70 \%$. Incorporation of ZDV prophylaxis into clinical practice, together with increased prenatal HIV counseling and testing, rapidly resulted in a significant decline in perinatal transmission and a concomitant decrease in the number of reported pediatric AIDS cases in the US. Transmission rates of 3-6\% have been reported in various cohort studies with ZDV prophylaxis alone, and of I-2\% when ZDV is combined with elective Cesarean delivery or when women are treated with highly active antiretroviral regimens that reduce maternal viral load to unquantifiable levels. Additionally, several short antiretroviral regimens, including those that require administration only during the intrapartum and early postpartum periods, have been shown to decrease perinatal transmission. These regimens provide effective intervention even for HIV-infected pregnant women who have not received antiretroviral therapy and are identified late in pregnancy or for the first time at delivery through rapid HIV testing.

However, this success has been partially offset by increasing HIV infection rates among young women, high adolescent pregnancy rates among at-risk populations, continued failure to identify HIV infection during pregnancy and inadequate prenatal care among HIV-infected women, particularly those using drugs. Additionally, the impact of evolving patterns of antiretroviral drug resistance on efficacy of prophylaxis is not known. As combination antiretroviral therapy becomes the standard of care for pregnant women in developed countries, evaluation of their infants for short- or long-term adverse consequences of intrauterine antiretroviral exposure is also a priority. Finally, clinical trials have identified short-course antiretroviral prophylaxis regimens that are effective and safe in resource-poor countries; however, transmission of HIV via breast milk remains a concern.

\section{Bibliography}

1. Centers for Disease Control. US Public Health Service Task Force recommendations for use of antiretroviral drugs during pregnancy for maternal health and reduction of perinatal transmission of human immunodeficiency virus type 1 in the United States. MMWR 2002;51 (Suppl. RR-18). Guidelines and drugs in pregnancy hyperlink are updated regularly and can be accessed on the HIV/AIDS Treatment Information Service website: http://AIDSInfo.nih.gov

2. Mofenson LM. Editorial: Tale of two epidemics the continuing challenge of preventing mother-to-child HIV transmission. J Infect Dis 2003;187:721-4

3. Watts DJ. Management of human immunodeficiency virus infection in pregnancy. N Engl J Med 2002;346: 1879-91

4. Minkoff H. Human immunodeficiency virus infection in pregnancy. Obstet Gynecol 2003;101:797-810

5. Mofenson LM. Committee on Pediatric AIDS. Technical report: perinatal human immunodeficiency virus testing and prevention of transmission. Pediatrics 2000;106(6): (http://www.pediatrics.org/cgi/content/full/ 106/6:e88) 
6. De Cock K, Fowler MG, Mercier E, et al. Prevention of mother-to-child HIV transmission in resource-poor countries: translating research into policy and practice. JAMA 2000;283:1175-82

7. Dabis F, Ekpini ER. HIV-1/AIDS and maternal and child health in Africa. Lancet 2002;359:2097-104

8. Nolan ML, Greenberg AE, Fowler MG. A review of clinical trials to prevent mother-to-child HIV-1 transmission in Africa and inform rational intervention strategies. AIDS 2002;16:1991-9

9. Mofenson LM, Munderi P. Safety of antiretroviral prophylaxis of perinatal transmission for HIV-infected pregnant women and their infants. JAIDS 2002;30:200-15

10. Nolan M, Fowler MG, Mofenson LM. Antiretroviral prophylaxis of perinatal HIV-1 transmission and the potential impact of antitretroviral resistance. JAIDS 2002;30:316-29

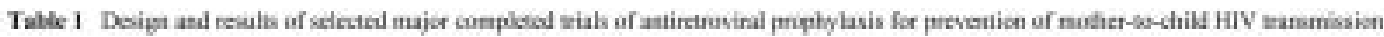

\begin{tabular}{|c|c|c|c|c|c|c|c|c|c|}
\hline \multirow[t]{3}{*}{ Trina' } & \multicolumn{5}{|c|}{ Drez aifvinismatiow no worher } & \multirow{2}{*}{\multicolumn{4}{|c|}{ 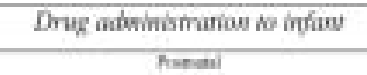 }} \\
\hline & \multicolumn{3}{|c|}{ Amprarat } & hropensen & \multirow{2}{*}{$\begin{array}{l}\text { Rominan } \\
\text { 1 wed }\end{array}$} & & & & \\
\hline & 14 ants & 3 क्ता & Wowt: & Lacabovm & & non & 19d & निता & Foods \\
\hline
\end{tabular}

Nen-

brasifectine

pusum

estrewn

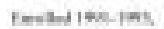

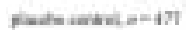

hropis, Thalind

tonationiar.

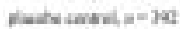

Pret, Bulad

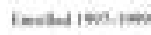

incascosits

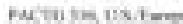
Busalanes" Frationseas

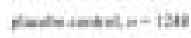
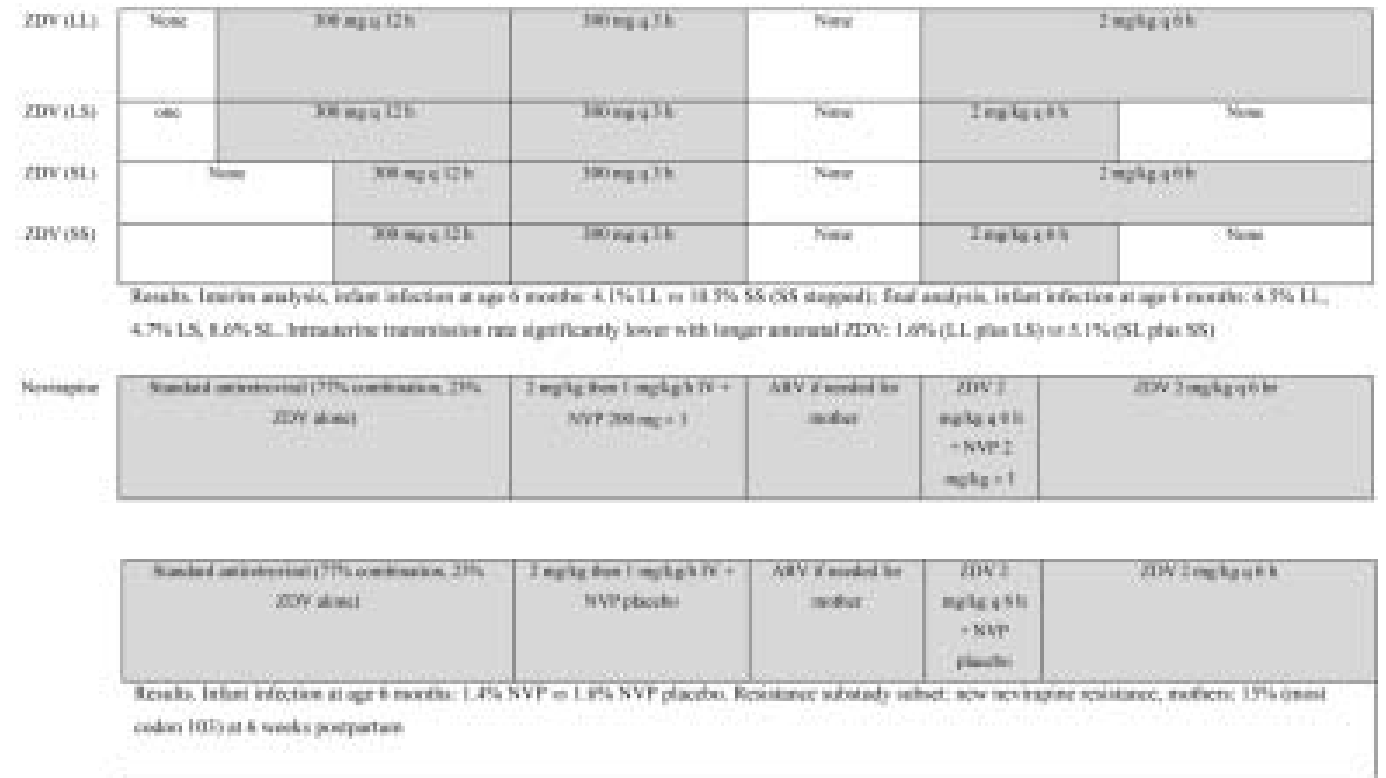

Table continued 
rial

\begin{tabular}{|c|c|c|c|c|c|c|c|c|}
\hline \multicolumn{5}{|c|}{ Drigg adwimistration to mather } & \multicolumn{4}{|c|}{ Dryg abininistinatiow wo infuw } \\
\hline & 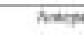 & & menten & Fonperion & & & andici & \\
\hline II $=$ and & is encels & Tant & Lenthm & inm & Esth & क्ष & $1 \mathrm{men}$ & $90 \mathrm{em}$ \\
\hline
\end{tabular}

ilecastfoeding

\begin{tabular}{|c|}
\hline 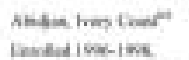 \\
\hline
\end{tabular}

now

ploctowered $1=20$

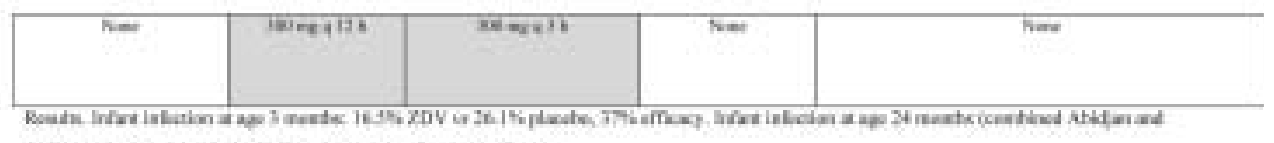

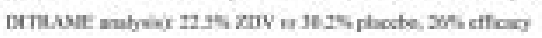

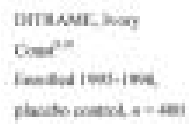

an

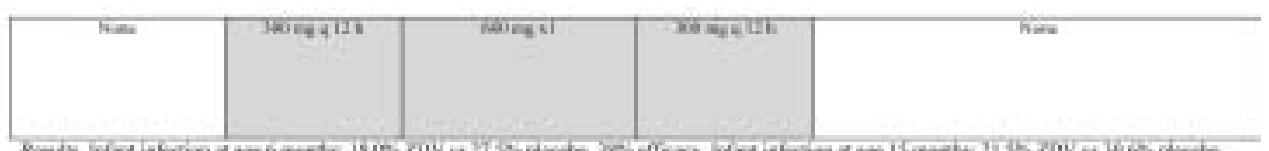

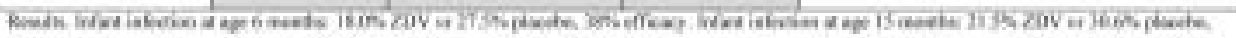

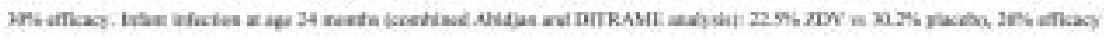

REma Anka indederis Dentad iverave Plachto enescl a -1 itr

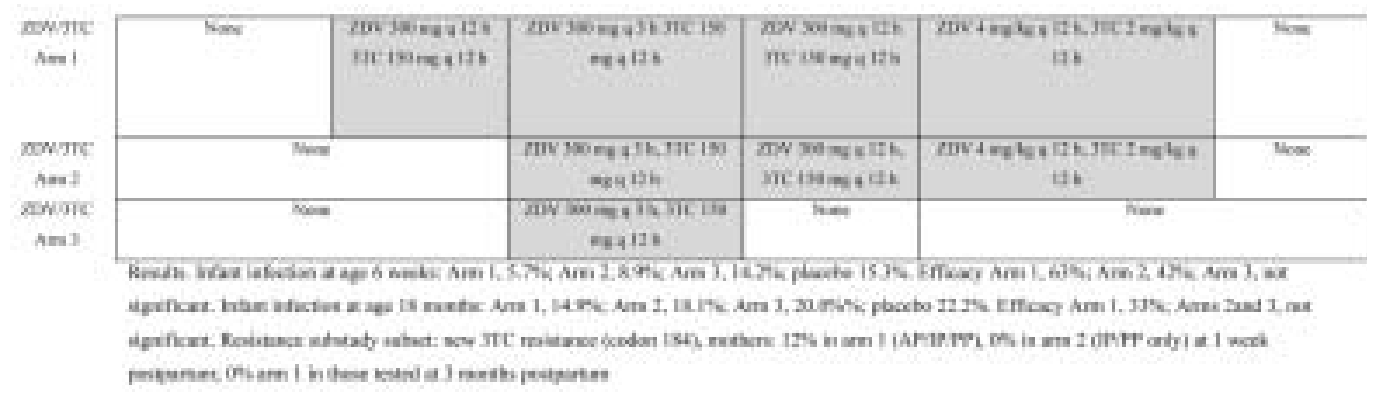

monrtas.

londient

Enodud ism.1nm.

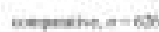

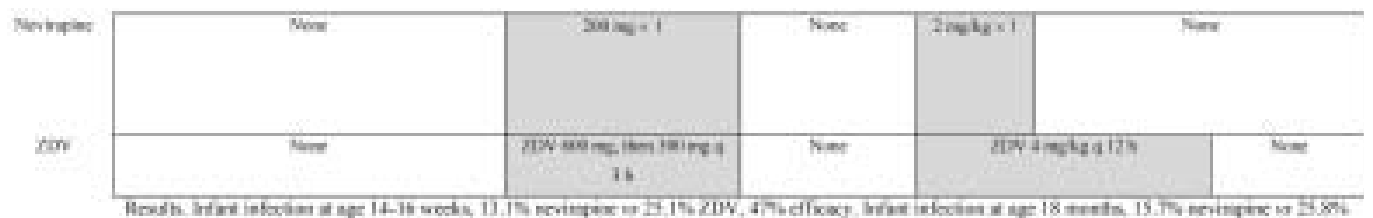

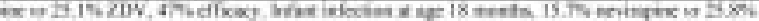

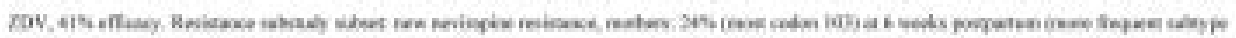

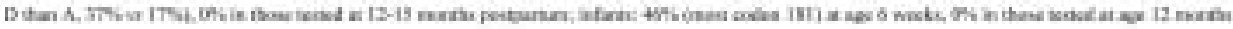

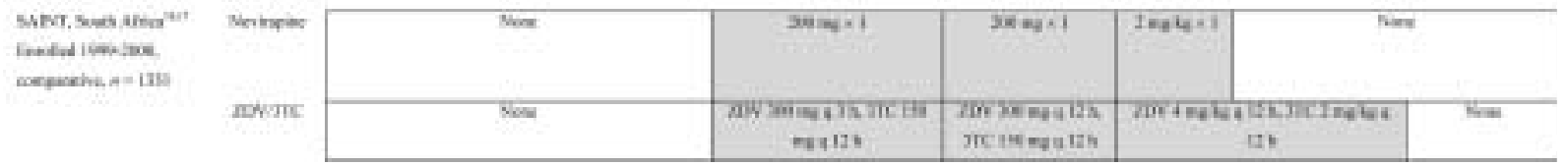

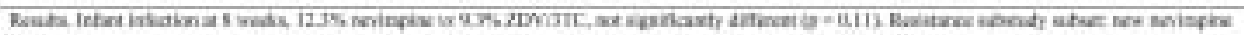

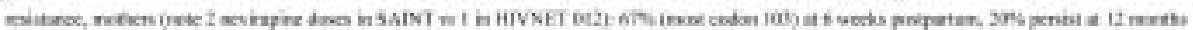

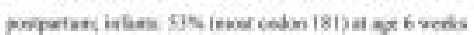

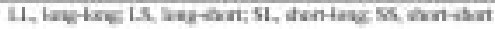

Table 2 Summary of US Public Health Service Task Force guidelines for prevention of mother-to-child HIV transmission in the US*

- Maternal antenatal plasma HIV RNA $\geqslant 1000$ copies $/ \mathrm{ml}$ :

- highly active antiretroviral therapy (HAART) (ideally containing ZDV after first trimester), plus ZDV intravenously intrapartum (HAART regimen continues during labor when possible) and ZDV for 6 weeks for infant. Maternal HAART can be stopped postpartum if therapy not required 
Table 2 (continued)

- elective Cesarean delivery if plasma HIV RNA remains > I,000 copies/ml near delivery

- Maternal plasma HIV RNA < I,000 copies/ml:

- ZDV antepartum after first trimester, intravenously intrapartum, and for 6 weeks for infant. Maternal ZDV discontinued after delivery

or

- $\quad$ highly active antiretroviral therapy (ideally containing ZDV after first trimester), plus ZDV intravenously intrapartum (HAART regimen continues during labor when possible) and ZDV for 6 weeks for infant. Maternal HAART can be stopped postpartum if therapy not required

- No antiretroviral therapy before labor:

- several effective regimens available to choose from for women who have had no previous therapy

- ZDV intravenously during labor, followed by 6 weeks of ZDV for infant

or

- ZDV/3TC orally during labor, followed by I week of ZDV/3TC for infant

or

- nevirapine, as single dose at onset of labor to mother, followed by single dose for newborn at age $48 \mathrm{~h}$. or

- intrapartum/postpartum ZDV regimen (above) plus single-dose nevirapine regimen (above)

- No antiretroviral therapy before or during labor:

- ZDV for 6 weeks for infant, started as soon as possible after delivery (preferably within 6-12 h of birth) or

- Some clinicians may choose to use ZDV in combination with additional antiretroviral drugs, but appropriate dosing regimens for neonates are incompletely defined and the additional efficacy of this approach in reducing transmission is theoretical but unproven

* To access the most recent guidelines, see: http://AIDSInfo.nih.gov

\section{References}

1. Connor EM, Sperling RS, Gelber R, et al. Reduction of maternal-infant transmission of human immunodeficiency virus type 1 with zidovudine treatment. N Engl J Med 1994;3312:1173-80

2. Eastman PS, Shapiro DE, Coombs RW, et al. Maternal viral genotypic zidovudine resistance and infrequent failure of zidovudine therapy to prevent perinatal transmission of human immunodeficiency virus type 1 in Pediatric AIDS Clinical Trials Group Protocol 076. J Infect Dis 1998;177:557-64

3. McSherry GD, Shapiro D, Coombs RW, et al. The effects of zidovudine in the subset of infants infected with human immunodeficiency virus type 1 (Pediatric AIDS Clinical Trials Group Protocol 076). J Pediatr 1999;717-24

4. Shaffer N, Chuachoowong PA, Mock C, et al. Short-course zidovudine for perinatal HIV-1 transmission in Bangkok, Thailand: a randomized controlled trial. Lancet 1999;353:773-80

5. Lallemant M, Jourdain G, Le Coeur S, et al. A trial of shortened zidovudine regimens to prevent mother-tochild transmission of human immunodeficiency virus type 1. N Engl J Med 2000;343:982-91

6. Dorenbaum A, Cunningham CK, Gelber RD, et al. Two-dose intrapartum/newborn nevirapine and standard antiretroviral therapy to reduce perinatal HIV-1 transmission: a randomized trial. JAMA 2002;288:189-98

7. Cunningham CK, Chaix M-L, Rekacewicz C, et al. Development of resistance mutations in women receiving standard antiretroviral therapy who received intrapartum nevirapine to prevent perinatal human immunodeficiency virus type 1 transmission: a substudy of Pediatric AIDS Clinical Trials Group protocol 316. J Infect Dis 2002;186:181-8

8. Wiktor S, Ekpini E, Karon J, et al. Short course oral zidovudine for prevention of mother-to-child transmission of HIV-1 in Abidjan, Cote d'Ivoire: a randomised trial. Lancet 1999;353:781-5. 
9. Leroy V, Karon JM, Alioum A, et al. Twenty-four month efficacy of a maternal short-course zidovudine regimen to prevent mother to child transmission of HIV-1 in West Africa. AIDS 2002;16:631-41

10. Dabis F, Msellati P, Meda N, et al. 6-month efficacy, tolerance, and acceptability of a short regimen of oral zidovudine to reduce vertical transmission of HIV in breastfed children in Cote d'Ivoire and Burkina Faso: a double-blind, placebo controlled multicentre trial. Lancet 1999;353:786-92

11. The Petra Study Team. Efficacy of three short-course regimens of zidovudine and lamivudine in preventing early and late transmission of HIV-1 from mother to child in Tanzania, South Africa, and Uganda (Petra study): a randomised, double-blind, placebo-controlled trial. Lancet 2002;359:1178-86

12. Giuliano M, Palmisano L, Galluzzo CM, et al. Selection of resistance mutations in pregnant women receiving zidovudine and lamivudine to prevent HIV perinatal transmission. AIDS 2003;17:1570-3

13. Guay L, Musoke P, Fleming T, et al. Intrapartum and neonatal single-dose nevirapine compared with zidovudine for prevention of mother-child transmission of HIV-1 in Kampala, Uganda: HIVNET 012 randomised trial. Lancet 1999;354:795-802

14. Eshleman SH, Mracna M, Guay L, et al. Selection and fading of resistance mutations in women and infants receiving nevirapine to prevent HIV-1 vertical transmission (HIVNET 012). AIDS 2001;15:1951-7

15. Eshleman SH, Guay LA, Mwatha A, et al. Extended analysis of nevirapine resistance in women with subtype A vs D HIV-1 6-8 weeks after single dose NVP prophylaxis: HIVNET 012. 10th Conference on Retroviruses and Opportunistic Infections, Boston, MA, February 10-14, 2003 (abs. 857).

16. Moodley D, Moodley J, Coovadia H, et al. The South African intrapartum nevirapine trial (SAINT): a multicenter, randomized, controlled trial of nevirapine compared to a combination of zidovudine and lamivudine to reduce intrapartum and early postpartum mother to child transmission of human immunodeficiency virus type-1. J Infect Dis 2003;187:725-35

17. Sullivan J. South African Intrapartum Nevirapine Trial: selection of resistance mutations. XIV International AIDS Conference, Barcelona, Spain, July 7-12, 2002 (abs. LBPeB9024) 


\title{
Oral presentations: session I
}

\section{Severe acute respiratory syndrome in an obstetric population}

\author{
S.F. Wong ${ }^{1}$, K.M. Chow ${ }^{1}$, L.C. Ho ${ }^{1}$, and B. Gonik ${ }^{2}$ \\ ${ }^{1}$ Princess Margaret Hospital, Kowloon, Hong Kong and ${ }^{2}$ Wayne State University, Detroit, Michigan
}

Objective: Severe acute respiratory syndrome (SARS) is a newly identified infectious disease with significant public health implications. At present no data are available regarding this disease in pregnancy. This report describes a series of SARS cases in pregnant women within a single healthcare facility.

Study design: This is a descriptive inpatient case series from the Princess Margaret Hospital in Hong Kong, a major infectious disease tertiary care facility in a designated epidemic region. SARS was identified in 7 pregnant women. The mean age was 31.0 years (range 22-34 years) with a gestational age range on admission of 6-3I weeks. Standard criteria were used to establish the probable diagnosis, including: epidemiologic linkage; clinical features of fever; flu-like symptoms; cough with progression to varying degrees of respiratory compromise; and the exclusion of other recognized pathogens.

Results: Probable coronavirus infection was confirmed by PCR in all subjects. Chest radiographs uniformly showed evidence of atypical pneumonia. The women were unresponsive to initial antimicrobial therapy, and changed to ribavirin and steroid therapy for deterioration in status 6-7 days after presentation. Mechanical ventilation was required in 3 cases. Cesarean delivery was undertaken for 2 women at 26 weeks and 31 weeks, respectively, for maternal deterioration, and for I woman at 28 weeks for suspected fetal compromise. The neonatal courses were normal and serial neonatal coronavirus PCRs were uniformly negative. In all, there were 2 maternal deaths and 2 women in the first trimester experienced spontaneous abortion.

Conclusion: Prevalence of the disease in pregnancy cannot be ascertained. Clinical presentation was similar to that in the non-gravid individual. The prognosis may be more guarded in pregnancy, with a high rate of ICU admissions (57\%), mechanical ventilation (43\%), and maternal death (29\%). At viability, there appears to be a lack of direct neonatal effect other than that associated with the maternal condition. Ribavirin and steroid therapy are controversial approaches to treatment in general, and are of particular concern in pregnancy. 


\title{
Severe acute respiratory syndrome (SARS) in pregnancy: a case report
}

\author{
M.H. Yudin ${ }^{1}$, D. Steele ${ }^{1}$, P. Kopplin ${ }^{2}$, M. Sgro ${ }^{3}$ and K. Gough ${ }^{2}$ \\ Departments of ${ }^{1}$ Obstetrics and Gynecology, ${ }^{2}$ Internal Medicine and ${ }^{3}$ Pediatrics, University of Toronto, \\ St. Michael's Hospital, Toronto, Canada
}

Objective: To describe a clinical case of severe acute respiratory syndrome (SARS) in pregnancy.

Study design: This is a case study of one patient, who was a 33-year-old $G_{1} P_{0}$ with a previously uncomplicated pregnancy. At 30 weeks' gestation she had direct contact with a family member who was subsequently diagnosed with probable SARS according to the World Health Organization case definition. While on home quarantine the subject of our study developed a fever of $38.0^{\circ}$, and was therefore admitted to hospital and isolated in a negative pressure room. She started therapy with azithromycin and cefuroxime but no antiviral medication.

Results: An initial chest radiograph demonstrated unilateral airspace disease. While in hospital, the subject experienced daily elevations in temperature and developed a dry cough, but with no shortness of breath. On the I I th day in hospital her chest radiograph demonstrated bilateral lung involvement, and she began intravenous steroid therapy. Over the next $48 \mathrm{~h}$ her temperature became normal, and she remained afebrile thereafter. Daily non-stress tests were performed and were always within normal limits. Ultrasound revealed a normally grown fetus with normal anatomy. There was increased amniotic fluid volume but no evidence of fetal hydrops or placentomegaly. Following discontinuation of steroids and antibiotics, the woman remained in hospital with no fever or respiratory symptoms for $48 \mathrm{~h}$, and was then discharged home. Cultures and serology for respiratory pathogens are negative to date. Her total time in hospital was 21 days, and she was at 34 weeks' gestation at discharge. The woman is currently still pregnant, and details regarding the remainder of pregnancy, labor and delivery, and postpartum and neonatal courses, will be provided at the annual scientific meeting.

Conclusion: Severe acute respiratory syndrome (SARS) is a new entity which is highly contagious and has an estimated $4 \%$ mortality rate. There is preliminary evidence that it may be associated with worse outcome when it occurs in pregnancy. It is still not known whether there are any long-term effects on fetuses when women are infected during pregnancy. In this case the patient responded well to antibiotics and steroids, without the use of antiviral medications. 


\title{
Normal neonatal outcome following West Nile virus (WNV) infection in the second trimester
}

\author{
M. Jelcz ${ }^{1}$, M.J. Dinsmoor ${ }^{1}$, K. Kaul ${ }^{2}$ and D. O’Leary ${ }^{3}$ \\ Departments of ${ }^{1}$ Obstetrics and Gynecology and ${ }^{2}$ Pathology, Evanston IL and the Arbovirus Disease Branch of \\ the Centers for Disease Control and ${ }^{3}$ Prevention, Fort Collins, CO, USA
}

Objectives: Fetal infection and severe central nervous system abnormalities have been reported following a case of intrauterine exposure to West Nile virus (WNV) in the third trimester of pregnancy (MMWR 2002;5 I: I I35-6). We describe a case of WNV acquired during the second trimester, that resulted in a normal neonatal outcome without evidence of congenital infection.

Study design: The patient was a 39-year-old $\mathrm{G}_{4} \mathrm{P}_{2}$ who presented in mid-August at 16 weeks' gestation, complaining of severe headache, photophobia and diffuse joint pains. Following diagnosis of acute WNV infection, the case was followed prospectively.

Results: Evaluation revealed that the woman was febrile $\left(38.5^{\circ} \mathrm{C}\right)$ with decreased range of motion of her neck. Analysis of cerebrospinal fluid (CSF) was consistent with aseptic meningitis (glucose $50 \mathrm{mg} / \mathrm{dl}$, protein $50 \mathrm{mg} / \mathrm{dl}, 3$ + WBC and no organisms on Gram stain). Serum and CSF were tested for arbovirus infection at a diagnostic reference laboratory. Acute WNV infection was confirmed, based on the presence in the CSF of WNV-specific antibodies at enzyme-linked immunosorbent assay and also by the presence in the serum of WNV-specific IgM and neutralizing antibodies. Level II ultrasound performed at 20, 24, and 31 weeks' gestation revealed no evidence of abnormalities and normal fetal growth. Following an uncomplicated labor at $39+$ weeks' gestation, the woman achieved the spontaneous vaginal delivery of an evidently normal girl weighing $3715 \mathrm{~g}$. Apgar scale 9 and 9. Intrauterine WNV infection of the infant was ruled out by the absence of WNV-specific IgM antibodies in umbilical cord plasma and the absence of WNV RNA (by TaqMan polymerase chain reaction) in umbilical cord plasma and tissue. Histopathologic studies of the placenta were normal. At 3 months of age the infant appears to be developing normally.

Conclusion: In this case, because of both the absence of WNV-specific antibodies and nucleic acid sequences in the infant's blood and the infant's normal development, it appears unlikely that transplacental WNV transmission occurred or that WNV meningoencephalitis during pregnancy had an adverse effect on the birth outcome. 


\title{
Parvovirus BI9 infection in pregnancy: lessons from a recent outbreak
}

\author{
H.N. Simhan and D.V. Landers \\ Department of Obstetrics, Gynecology and Reproductive Sciences, University of Pittsburgh, PA, USA
}

Objectives: Parvovirus BI9 (Parvo) infection in pregnancy has long been recognized as a cause of fetal compromise and mortality. Our goal was to describe our experience with Parvo infection in pregnancy with respect to seasonal and annual variation, geographic distribution, clinical presentation and neonatal outcome. We hypothesize that, contrary to published evidence, the majority of women who present with documented maternal Parvo are asymptomatic.

Study design: This was a cohort study of all women at Magee-Women's Hospital with serologically documented Parvo during pregnancy, from 1998 to 2001 . The medical records of all such women were reviewed to gather demographic, clinical, sonographic, test and outcome data.

Results: From 1998 to 200I, 25 cases of Parvo infection during pregnancy were serologically documented. There was a clear seasonal variation in disease incidence, with all cases occurring from January to June. The frequency of cases varied substantially from year to year as well, with 14 cases in 1998, 0 cases in 1999 and 2000, and II cases in 200I. The cases occurring in Allegheny County were geographically clustered in school district patterns. Only 4 (16\%) women with documented infection experienced any symptoms. There were 3 cases (I2\%) of hydrops and 4 cases $(16 \%)$ of fetal death. We noted spontaneous resolution of I case of hydrops, and I case of fetal death occurred despite reassuring sonographic testing within I week.

Conclusion: Our experience with Parvo in pregnancy confirms the seasonal and annual variation in disease frequency. The frequency and severity of maternal symptoms appear to be less than those reported in the literature. The case of spontaneous resolution of hydrops and the case of rapid fetal decompensation point to potential problems with current management and counseling strategies. 


\title{
Placental histopathology in spontaneous versus medically indicated preterm birth
}

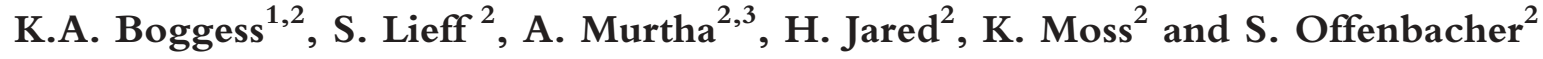 \\ ${ }^{1}$ Department of Obstetrics and Gynecology and ${ }^{2}$ Center for Oral and Systemic Diseases, UNC at Chapel Hill, \\ and ${ }^{3}$ Department of Obstetrics and Gynecology, Duke University
}

This study was supported by NIDCR R01-DE-12453 and HD K12 01441.

Objective: To determine whether placental histopathology differs with type of preterm birth.

Study design: A prospective cohort of pregnant women who delivered between $20^{\circ}$ and $36^{6}$ weeks' gestation was used for this analysis. Type of preterm birth was considered spontaneous (SP) if preterm labor (PTL) or PROM occurred, and medically indicated (IND) if there was no PTL or PROM and delivery was induced. Placental histopathology was examined to determine presence of inflammation (chorioamnionitis, funisitis), vasculopathy and infarction. Chi-square and logistic regression analyses were used and $p$ value $<0.05$ was considered significant.

Results: Of 1067 women enrolled in the study, 180 (16.9\%) delivered preterm, i.e. 124 at 34-36 weeks, 22 at $32-33^{6}$ weeks, 18 at $28-31^{6}$ weeks, 12 at $23-27^{6}$ weeks and 4 at $20-22^{6}$. weeks of gestation. In all $107(59.4 \%)$ were SP and 73 (40.6\%) were IND. Of the SP births, 39 (36.4\%) were due to PROM and 60 (56. I\%) were due to PTL. The most common reason for IND birth was pre-eclampsia. Placental pathology findings were available for 103 cases (56 SP and 47 IND), as in Table I.

Table I Placental histopathology by delivery type

\begin{tabular}{lccc}
\hline Placental histopathology & SP preterm birth $(n=56)$ & IND preterm birth $(n=47)$ & $p$ Value \\
\hline Chorioamnionitis, any & $31(55.4 \%)$ & $14(29.8 \%)$ & 0.009 \\
Chorioamnionitis, severe & $6(10.7 \%)$ & 0 & 0.019 \\
Funisitis & $11(19.6 \%)$ & $2(4.3 \%)$ & 0.022 \\
Vasculopathy & $2(3.6 \%)$ & $8(17 \%)$ & 0.015 \\
Infarction & 3 & $10(21.3 \%)$ & \\
\hline
\end{tabular}

SP, spontaneous; IND, medically indicated

Gestational age was significantly lower in SP than in IND births $(p=0.002)$. After controlling for gestational age, the risk for placental inflammation remained significantly increased in SP compared with IND preterm births (odds ratio $2.4,95 \%$ confidence interval I.I-5.7).

Conclusion: Among preterm births, placental inflammation occurred more commonly in spontaneous cases and placental vasculopathy more commonly in medically indicated cases. This suggests that type of preterm birth should be considered when studying prematurity, as placental effects may differ. 


\title{
Oral presentations: session II
}

\section{HIV-I virologic failure may occur earlier in the blood plasma than in the genital tract of infected women receiving antiretroviral therapy}

\author{
S. Cu-Uvin, A. Caliendo, B. Snyder, J. Hogan, K. Mayer, S. Donahue, J. Ingersoll, \\ J. Kurpewski, J. Harwell, T. Flanigan and C.C.J. Carpenter
}

Objective: We sought to determine whether virologic failure occurs earlier in the blood or in the genital tract of women who are being prospectively followed during successful antiretroviral therapy.

Study design: The study involved $47 \mathrm{HIV}$-infected women who achieved below detectable PVL and cervicovaginal lavage (CVL) viral load after initiation of highly active antiretroviral therapy (HAART). On follow-up, a PVL or CVL viral load $>400$ copies $/ \mathrm{ml}$ (if the preceding PVL or CVL viral load was $<400$ copies $/ \mathrm{ml}$ ) was considered a viral breakthrough. Paired PVL and CVL viral load was estimated at baseline, 2 weeks, I month, and every 6 months after starting HAART. HIV-I RNA was quantified by NASBA (lower limit of detection at 400 copies/ml).

Results: Of the 47 subjects, $66 \%$ were antiretroviral-naive and $34 \%$ were nucleoside-experienced before initiation of HAART. Nucleosides were with either protease inhibitor or NNRTI. At baseline, $42 / 47$ (89\%) had detectable PVL (mean PVL: 108426 copies/ml) and I3/47 (28\%) had detectable CVL HIV-IRNA (mean CVL viral load: 291396 copies $/ \mathrm{ml}$ ). On follow-up, 23 women maintained PVL and CVL viral load at $<400$ copies/ml at the same time points, and 24 women had viral breakthrough in blood plasma or genital tract or both. During viral breakthrough, I4/24 (58\%) had PVL >400 copies/ml (mean: 16 906; range: 670-46 000 copies/ml) but had below detectable HIV-I RNA in the genital tract. At the same time point 10/24 (42\%) had detectable HIV-I RNA in both blood plasma (mean: 38 370; range: I 400-200 000 copies/ml) and CVL (mean: 39 760; range: I I00-I70 000 copies $/ \mathrm{ml}$ ). During viral breakthrough, no woman had a detectable CVL viral load when the PVL was $<400$ copies/ml HIV-I RNA.

Conclusion: HAART suppressed virus in both blood plasma and genital tract of HIV-infected women. However, when therapy failed, viral rebound occurred more often and earlier in the blood plasma compartment compared with the genital tract compartment. 


\title{
Drug-selected HIV-I mutations can differ in cervicovaginal and blood plasma RNA
}

\author{
M.P. DePasquale, J. Allega, L. Sutton, R.T. D’Aquila, A.C. Caliendo, S. Donahue, \\ and $\mathrm{S}$. Cu-Uvin
}

Objective: To compare replication of drug-selected HIV-I genotypes in the female genital tract and blood plasma.

Study design: We studied 7 HIV-positive women who were receiving failing ART and 3 HIV-positive untreated women. Each had detectable viral load in plasma and cervico-vaginal lavage (CVL) or in endocervical secretions collected by Sno-strips (Nuclisens). RNA extracted (Qiagen) from plasma and either CVL or Sno-strips was examined by RT PCR. Population sequence was determined with standard HIV pol cycle sequencing kits ( 9 VGI, I $A B I)$. HIV DNA from cells centrifuged from CVL was also sequenced using 3 of the treated women's specimens.

Results: Of the 7 women on a failing antiretroviral regimen, 5 had drug-selected mutations in cervicovaginal RNA which were not detected in the plasma, including mutations in PR (46I, 54V, 82A, 84V, 90M) and RT (I84V). Other sequence differences were also seen between plasma and CVL. One CVL sequence was quite diverse and diverged markedly from the corresponding plasma sequence. Of the 7 women receiving failing therapy, 2 had the same genotype in plasma and genital secretions; I had multiple PR and RT mutations in both fluids; and I had no mutations in either fluid. CVL cell DNA sequences were probably archival, relative to CVL RNA, in 2 of the 3 ART failures. In contrast, sequences from each of 3 drug-naive women's plasma and CVL did not differ in any drug-selected positions; polymorphisms and silent mutations at other positions were also similar.

Conclusion: Drug-selected mutations in cervicovaginal secretions can differ from those dominant in the blood during ART failure. Further studies are needed to define the implications for vertical or heterosexual transmission of resistant virus. 


\title{
Seroprevalence rate of HIV-I in women in labor with no prenatal care at a community hospital in Newark, New Jersey, using expedited ELISA testing
}

\author{
A. Al-Khan, A. Bardeguez, B. Cicuto and J. Apuzzio \\ University of Medicine and Dentistry of New Jersey, New Jersey Medical School Department of Obstetrics, \\ Gynecology and Woman's Health, Division of Maternal Fetal Medicine, Columbus Hospital, Newark, \\ New Jersey.
}

Objective: In all, $40 \%$ of women infected with HIV-I are not diagnosed until after delivery. The recent introduction of rapid testing algorithms has been used to determine HIV seropositivity in pregnant women receiving no prenatal care, hoping to reduce perinatal transmission by aggressive medical intervention. The purpose of this study was to determine the HIV seropositivity rate in women with no prenatal care using a protocol including an expedited ELISA.

Study design: A retrospective chart analysis was completed from January 202002 to December 3 I 2002. HIV testing of women receiving prenatal care was carried out using ELISA and positive tests were confirmed by Western blot. Expedited ELISA was performed for women receiving no prenatal care and reported within I $\mathrm{h}$ from the time the blood specimens were obtained. Positive expedited ELISA results were later confirmed using Western blot.

Results: During the study period there were 1342 live births, including 56 mothers (4. $17 \%$ ) who had received no prenatal care. Of the 1286 mothers with prenatal care, II (0.86\%) were seropositive for HIV-I. Of the 56 mothers with no prenatal care, 2 (3.6\%) were HIV-positive by ELISA and both results were subsequently confirmed by Western blot.

Conclusion: The seroprevalance rate for HIV-I was about four times higher in women receiving no prenatal care. A protocol using expedited ELISA HIV testing was useful in identifying HIV-positive patients in order to offer intrapartum antiretroviral therapy to decrease perinatal transmission. 


\title{
Outcomes associated with ART/HAART use in HIV-infected pregnant women
}

\author{
D. Money, J. Forbes, K. Craib, M. Math, G.P. Meneilly, D. Khoo, D. Bloomenthal, \\ A. Alimenti and D. Burdge \\ Oak Tree Clinic, Children's and Women's Health Centre of British Columbia, and Departments of Obstetrics \\ and Gynecology, Paediatrics and Medicine, University of British Columbia
}

Objectives: To examine pregnancy and maternity outcomes in a cohort of HIV-infected women on antiretroviral therapy during pregnancy.

Study design: This was a cohort study of HIV-infected pregnant women and infants followed from 1990 to 2002, through a tertiary referral centre for women and children in British Columbia, Canada.

Results: We reviewed 282 pregnancies that resulted in 247 live births, I I spontaneous abortions, 22 terminations of pregnancy and 2 stillbirths. In 152 of the 247 pregnancies resulting in live births (in 218 women), the mother received some antiretroviral therapy. The antenatal ART therapy (for $>2$ weeks) included 24 women receiving AZT, 12 receiving dual nucleosides, 87 receiving HAART and 29 who received intrapartum ART alone. Of the women receiving HAART, I received a triple nucleoside regimen, 42 a regimen based on protease inhibitor (PI), 42 non-nucleoside (nevirapine) regimens of which 4 changed to $\mathrm{PI}$ in later pregnancy, and 2 received multidrug salvage regimens. Overall, changes in HAART drug combinations during pregnancy were made in 14 cases (16\%). These were due to toxicity in $4 / 87(4.5 \%)$ and to inadequate initial virologic response in 10/87 (1I.5\%). Antenatal obstetric complications in this cohort included IUGR (5.2\%), preterm labour (3.2\%), gestational diabetes (3.2\%), hyperemesis gravidarum (2.6\%), oligohydramnios (1.9\%), polyhydramnios (1.9\%), $\mathrm{PIH}(1.3 \%)$, and antepartum hemorrhage $(0.6 \%)$. Preterm birth $(<37 \mathrm{wks}$ ) occurred in $22.6 \%$. Preliminary analysis showed a trend to higher rates of significant toxicity (rash, hyperbilirubinemia, fevers) in women receiving NNRTI (4/42) compared with women receiving $\mathrm{PI}(0 / 42, \mathrm{p}=0.116)$. For those pregnancies in which the women received antenatal ART, the vertical transmission rate was $0 \%$.

Conclusion: HIV-infected pregnant women generally tolerate combination antiretroviral therapy well, with marked reduction in vertical transmission ( $0 \%$ in this study). However, further investigation is required to evaluate potential toxicity and efficacy differences between regimens based on PI compared with those based on NNRTI. 


\title{
Factors associated with HIV-I RNA shedding in the female genital tract: 24 months' follow-up
}

\author{
S. Cu-Uvin, A. Caliendo, B. Snyder, J. Hogan, K. Mayer, S. Donahue, J. Allega, \\ J. Kurpewski, J. Harwell, T. Flanigan and C.C.J. Carpenter
}

Objective: We aimed to determine the association between cervicovaginal lavage (CVL) HIV-I RNA and antiretroviral therapy, PVL, CD4 count, lower genital tract infections (LGTI) and the presence of semen among HIV-infected women followed over a 24-month period.

Study design: To assess the effect of antiretroviral therapy on HIV-I in the genital tract, 97 HIV-infected women were enrolled in a longitudinal study. PVL, CVL viral load and tests for presence of LGTI and semen were done at baseline, 2 weeks, I month and every 6 months thereafter. HIV-I RNA was quantified by NASBA. The subjects were divided into 3 groups: group A comprised 36 women who were antiretroviral-naïve and were starting HAART; group B included 26 women who were nucleoside-experienced and starting HAART; and the 35 women in group $C$ had received no past or present therapy.

Results: Of the 97 subjects, $68 \%$ had had sexual intercourse with a known HIV-positive partner. None of the women had GC, chlamydia or SY; $6 \%$ of the samples were positive for trichomonas, $35 \%$ for BV, $18 \%$ for candida and $6 \%$ for semen (Table I).

On logistic regression analysis, there was a highly significant association between PVL and detection of CVL HIV-I

Table I Percentage of CVL HIV-IRNA $<400$ copies/ml by group over all follow-up visits

\begin{tabular}{lccccccc}
\hline Group & Baseline & $\mathbf{2}$ wks & $\mathbf{I}$ mo & $\mathbf{6}$ mos & $\mathbf{1 2}$ mos & $\mathbf{1 8}$ mos & $\mathbf{2 4}$ mos \\
\hline A & $6 \%$ & $83 \%$ & $86 \%$ & $92 \%$ & $88 \%$ & $88 \%$ & $83 \%$ \\
B & $71 \%$ & $78 \%$ & $82 \%$ & $73 \%$ & $93 \%$ & $90 \%$ & $91 \%$ \\
C & $79 \%$ & $92 \%$ & $81 \%$ & $79 \%$ & $75 \%$ & $84 \%$ & $61 \%$ \\
\hline
\end{tabular}

wk, week; mo, month

RNA. For each $\log _{10}$ increase in PVL, there was a fourfold increase in the odds of CVL viral load detection (OR $37, p<000 \mathrm{I})$. The presence of semen in the genital tract was significantly associated with detectable CVL viral load (OR 2.3, $p=0.39$ ). There was no significant difference between CVL HIV-I RNA detection by antiretroviral grouping and by CD4 cell count.

Conclusion: Plasma viral load was most associated with the presence of HIV-I RNA in the genital tract. Many HIV-positive women had HIV-positive partners. We conclude that genital tract samples should be investigated for the presence of semen because this was significantly associated with HIV-I in the female genital tract and the source of HIV-I may be from an HIV-positive partner. 


\title{
Oral presentations: session III
}

\section{One additional dose of antibiotics is sufficient postpartum therapy for chorioamnionitis}

\author{
R.K. Edwards and P. Duff
}

Objective: To determine whether a short course of antibiotics (intrapartum plus one dose postpartum) provides adequate treatment for chorioamnionitis.

Study design: In this randomized clinical trial, women received intravenous ampicillin, $2 \mathrm{~g}$ every $6 \mathrm{~h}$, and gentamicin, $1.5 \mathrm{mg} / \mathrm{kg}$ every $8 \mathrm{~h}$, when clinical chorioamnionitis was diagnosed. If delivered by Cesarean section, mothers received clindamycin, $900 \mathrm{mg}$ intravenously, at the time of umbilical cord clamping. The control group continued to receive clindamycin every $8 \mathrm{~h}$ until afebrile and asymptomatic for at least $24 \mathrm{~h}$. The study group received no additional doses of clindamycin. The primary outcome was treatment failure, defined as body temperature reading after the first postpartum dose of antibiotics, either once above $39.0^{\circ} \mathrm{C}$, or twice above $38.4^{\circ} \mathrm{C}$ at least $4 \mathrm{~h}$ apart. These subjects received additional antibiotics. Secondary outcome variables included duration of hospital stay, antibiotic doses received, and infection-related complications. To detect a $150 \%$ increase in the rate of treatment failures from $8 \%$ in the control group to $20 \%$ in the study group, 292 subjects were needed $(\alpha=0.05 ; \mathrm{I}-\beta=0.80)$.

Results: In the intent-to-treat analysis, treatment failure rate did not differ between the control $(n=14 I)$ and study $(n=15 \mathrm{I})$ groups overall (3.5\% vs $4.6 \% ; p=0.639)$, for vaginal deliveries ( $4.5 \%$ vs $3.4 \%$; $p=0.999)$, or for Cesarean deliveries ( $1.9 \%$ vs $6.3 \% ; p=0.375)$. Women in the control group received more antibiotic doses postpartum $(17.7+8.4$ vs $3.7+6.0 ; p<000 \mathrm{I})$ and during the intrapartum and postpartum period $(20.7+8.6 \mathrm{vs}$ $6.2+4.8 ; p<0.000 \mathrm{I})$. A larger proportion of women in the control group remained in hospital after the second postpartum day if delivered vaginally $(14.8 \%$ vs $6.9 \% ; p=0.049)$ and after the third postoperative day if delivered by Cesarean section $(20.8 \%$ vs $10.9 \% ; p=0.143)$. Infection-related complications were rare and did not differ between groups.

Conclusion: One additional dose of a broad spectrum combination of antibiotics was sufficient postpartum therapy for women with chorioamnionitis. 


\title{
Elevated cytokines associated with post-Cesarean endometritis
}

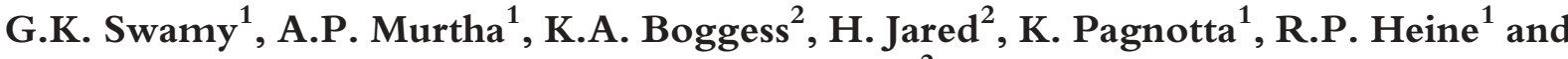 \\ S. Offenbacher ${ }^{2}$ \\ ${ }^{1}$ Duke University Medical Center, Durham, NC, USA and ${ }^{2}$ School of Dentistry, University of North \\ Carolina, Chapel Hill, NC, USA
}

This study was supported by RO-1-DE-12453.

Objective: Elevated cytokine levels are seen in the setting of active infection. It is unclear whether elevated cytokine levels are detectable before the onset of infection as evidence of impending disease. Our objective was to determine whether cytokine levels are elevated before the development of post-Cesarean endometritis.

Study design: The Oral Conditions and Pregnancy study was designed to examine the relationship of adverse pregnancy outcomes to periodontal disease. Maternal serum was obtained before 26 weeks' gestation and again upon hospital admission for delivery. Maternal serum was subsequently analyzed for C-reactive protein (CRP), interleukin(IL)-6, IL-I $\beta$, soluble intercellular adhesion molecule-I (sICAM-I), and tumor necrosis factor- $\alpha$ (TNF$\alpha)$ using ELISA. Subjects were divided into those with and without postoperative endometritis. Endometritis was defined as temperature $\geqslant 38.0^{\circ} \mathrm{C}$ on or after postoperative day 2 , with uterine tenderness. Data were analyzed using Student's $t$-test and Mann-Whitney U-test (SAS 8.2, Cary, NC).

Results: Of the 1019 women enrolled, 207 (21.3\%) underwent Cesarean delivery and approximately 9\% (20/ 207) had endometritis. There was no difference in age, marital status, race, weight, tobacco use or labor before Cesarean delivery between groups. Women with endometritis were more likely to have Medicaid insurance $(p=0.05)$. Median CRP upon admission was significantly higher in women with endometritis $(14.27 \mathrm{vs} 6.7 \mu \mathrm{g} / \mathrm{ml}$, $p=0.04)$. The same association was detected in mean sICAM-I levels upon admission (4I6.l I vs $316.3 \mathrm{ng} / \mathrm{ml}$, $p=0.005)$. There was no difference in IL-I $\beta$, IL- 6 or TNF- $\alpha$ between the two groups.

Conclusion: Measurement of maternal serum CRP and sICAM-I upon hospital admission may identify those women at higher risk for postoperative endometritis. 


\title{
Subclinical endometritis as a potential etiologic agent for idiopathic preterm birth: a pilot study
}

\author{
S. Gopalani, L.O. Eckert, K. Agnew, N. Kiviat and D. Eschenbach
}

Objective: Whereas the association of intra-amniotic bacterial colonization with idiopathic preterm birth has been well documented, its temporal relationship has not. We hypothesize that women with a previous preterm birth have an increased prevalence of subclinical endometritis in the non-pregnant state, compared with women with previous term births.

Study design: This was a nested cross-sectional pilot study of the association of subclinical endometritis and endometrial colonization with a history of preterm birth (defined as occurring between 12 and 36.9 weeks' gestation). Cases $(n=35)$ were selected if they had at least one preterm birth, and controls $(n=73)$ had term births only. Exclusion criteria were age greater than 35 years or less than 16 years, recent antibiotic therapy, and clinical signs of pelvic inflammatory disease. A portion of endometrial tissue was used for histologic evaluation and for aerobic and anaerobic culture. Histologic endometritis was defined as $\geqslant 5$ neutrophils $/ 400 \times$ field in the epithelial surface, with $\geqslant I$ plasma cell per $120 \times$ field in the endometrial stroma. Statistical analysis was by Fisher's exact test or Pearson's chi-square test, as appropriate.

Results: There was no significant difference in either the prevalence of subclinical endometritis ( $14.3 \% \mathrm{vs} 27.1 \%$, $p=0.10)$ or upper genital tract colonization with pathogenic flora $(45.7 \%$ vs $38.6 \%, p=0.3)$ between cases and controls. The prevalence of infection with $N$. gonorrhoae or C. trachomatis, as well as history of previous PID, was similar between both groups. Women with a history of previous preterm birth had a trend towards higher risk of mucopurulent cervicitis compared with controls ( $31.4 \%$ vs $15.7 \%, p=0.06)$.

Conclusion: In this pilot study, we did not detect a cross-sectional temporal association between subclinical endometritis in the non-pregnant state and a history of preterm birth. Future investigations should examine the relationship between subclinical endometritis and prematurity in a longitudinal fashion. 


\title{
Early but not delayed antibiotic treatment prevents preterm delivery following experimental intra-amniotic infection
}

\author{
M.G. Gravett, D.W. Sadowsky, S.S. Witkin and M.J. Novy \\ Reproductive Sciences, Oregon National Primate Research Center OB/GYN, Oregon Health Sciences \\ University Weill Medical College
}

Objective: Preterm birth associated with intra-amniotic infection (IAI) is a major cause of extreme prematurity. Antibiotics, however, have not been effective in preventing infection-induced prematurity. We studied chronically instrumented pregnant rhesus monkeys to examine reasons for failure of antibiotics to prevent preterm delivery in the setting of IAI.

Study design: A total of 12 chronically instrumented pregnant rhesus monkeys with timed gestations received experimental IAI by intra-amniotic inoculation of 106 Group B streptococci (GBS) at I34 to I4I days of gestation; term is 167 days. Amniotic fluid (AF) cytokines (IL-I beta, IL-6, IL-8, TNF-alpha), prostaglandins (PGE-2 and PGF2alpha), matrix metalloproteinases (MMP-2, MMP-9), and uterine contractility (hourly contraction area in $\mathrm{mmHg}$ / $\mathrm{s} / \mathrm{h}$ ) were serially measured before and after infection. Antibiotics (ampicillin $15 \mathrm{mg} / \mathrm{kg}$ maternal IV every $6 \mathrm{~h}$ ) were given to 2 animals $8 \mathrm{~h}$ after inoculation and before increases in uterine contractility; 4 animals received ampicillin after the onset of uterine contractions; and 6 animals received no antibiotics (control group).

Results: AF bacteria were successfully eradicated from animals treated $8 \mathrm{~h}$ after inoculation and no increases in uterine contractility or in AF cytokines or prostaglandins were observed. In contrast, animals receiving antibiotics after the onset of contractions showed increased AF cytokines and prostaglandins $(p<0.05$ versus early treatment) and in MMP-9 $93 \mathrm{kD}$ proenzyme and $82 \mathrm{kD}$ active enzyme, which persisted until preterm delivery despite successful eradication of GBS from the AF and from the fetus. The mean inoculation to delivery interval was $8 \mathrm{I} \mathrm{h}$ in animals receiving antibiotics after the onset of contractions and was not significantly different from the mean interval of $37 \mathrm{~h}$ in the 6 animals receiving no antibiotics $(p=0.2)$.

Conclusion: Antibiotic treatment begun before the onset of uterine contractility eliminated AF GBS and prevented elevations in AF cytokines, prostaglandins, and preterm delivery. Antibiotics given after the onset of uterine contractions did not prevent preterm delivery despite elimination of GBS from the AF. These data support the central role of the proinflammatory response in infection-induced preterm birth and suggest that novel immunomodulators in combination with antibiotics may be more effective than antibiotic therapy alone, after the onset of contractions, in the prevention of infection-induced preterm birth. These data also point to the need for sensitive diagnostic tests for the early detection of IAI.

Supported by NIH AI42490, HD06159 


\title{
Single nucleotide polymorphisms are associated with chorioamnionitis in preterm labor and preterm premature rupture of membranes
}

\author{
G.K. Swamy, A.P. Murtha, T.S. Smith, M. Betts-Sonstegard, B. Yonish and \\ R.P. Heine \\ Duke University Medical Center, Durham, NC, USA
}

Objective: Infection in the setting of preterm premature rupture of membranes (PPROM) and preterm labor (PTL) continues to be a leading cause of neonatal morbidity and mortality. We previously demonstrated that maternal serum cytokines were elevated in women with chorioamnionitis. Our objective was to determine whether single nucleotide polymorphisms (SNP) in pro- and anti-inflammatory cytokines were associated with chorioamnionitis in women with PPROM or PTL.

Study design: All patients with PPROM or PTL at less than 34 weeks' gestation were eligible for enrollment. Maternal and cord blood was obtained for DNA extraction. Genotyping was determined using PCR and restriction enzyme digest for the IL-6-174, IL-6-597, IL- I0-592, IL- IL-1082 and TNF alpha-863 SNPs. Placental pathology was determined by a staff pathologist. Women were divided into those with and those without chorioamnionitis. Data were analyzed using Fisher's exact test and Mann-Whitney's U-test (SAS 8.2, Cary, NC). Results: Of the 18 subjects enrolled, 7 (39\%) had chorioamnionitis and II (6I\%) had no chorioamnionitis. There was no difference in mean maternal age or race between groups. IL-6- I74 and IL-6-597 appear to be linked, such that all samples homozygous for $G$ at -174 were homozygous for $G$ at -597 . Those that were heterozygous $G / C$ at -174 were heterozygous $G / A$ at -597 . The homozygous $C / C$ variant (-174) and $A / A$ variant (-597) were not present in our sample. Patients with chorioamnionitis were more likely to have the heterozygous variant than those without chorioamnionitis ( $83.3 \%$ vs $16.7 \%, p=0.01$ ). There was no significant difference in the remaining polymorphisms.

Conclusion: Polymorphisms in the IL-6 gene promoter region may be associated with an increased risk of chorioamnionitis in PPROM and PTL. Such variations may impact on the morbidity associated with preterm birth. 


\title{
Oral presentations: session IV
}

\section{Preterm intrauterine infection and immunoregulatory gene polymorphisms}

\author{
H.M. McDonald ${ }^{1}$, M.F. Annells ${ }^{1}$, P.H. Hart ${ }^{2}$, S. Heatley ${ }^{3}$ and C.G. Mullighan ${ }^{1,3}$ \\ ${ }^{1}$ Women's Children's Hospital, ${ }^{2}$ Flinders University and ${ }^{3}$ Australian Red Cross Blood Services (SA), \\ Adelaide, Australia
}

Objective: Genetic differences in immunoregulators may influence a woman's susceptibility to intrauterine infection (IUI) and subsequent preterm birth. The aim of this study was to examine the association(s) between maternal carriage of certain gene polymorphisms in immunomodulatory regulators and IUI.

Study design: Blood was collected from 202 Caucasian women with spontaneous preterm labor and delivery before 35 weeks' gestation; $8 \mathrm{I}$ women had histologic evidence of IUI (defined as chorioamnionitis, decidualitis, villitis or funisitis) and these were compared with 100 women with negative histology (2I had no placental histology performed). DNA was extracted and genotyped by PCR using published sequence specific primers. Frequencies of single nucleotide polymorphisms (SNPs) were studied for IL-I $\alpha$, IL-IR receptor I, IL-I receptor antagonist, IL-4, IL-6 (each at one position); IL-I $\beta$, TGF $\beta$ I, Fas, mannose binding lectin (each at 2 positions); and IL- 10 and TNF $\alpha$ (each at 3 positions).

Results: Most SNPs tested had similar frequencies in women with and without IUI. However, Fas frequencies of the high-producing allele $G$ at $-1377(0.91$ vs 0.86 , OR I.8, $p=0.09)$ and corresponding $G / G$ genotype were higher in IUI-positive women (G/G 0.85 vs 0.73 , OR 2.I, Cl I.0-5.0, $p=0.05$ ). There were also differences in carriage of the high-producing TGF $\beta-509$ T allele $(0.24$ vs $0.32, p=0.12)$ and T/T genotype $(0.04$ vs 0.13, OR 0.3 , $\mathrm{Cl} 0.1-0.99, p=0.03$ ) between the IUI-positive and IUI-negative women.

Conclusion: The etiology of intrauterine infection may be influenced by imunoregulatory SNPs, such as Fasmediated apoptosis, other than those contributing to preterm birth with no evidence of infection. 


\title{
Identification of novel peptides in intra-amniotic infection by proteomics
}

\author{
M.G. Gravett, S.R. Nagalla, R.G. Rosenfeld, J. Hitti, D.A. Eschenbach and M.J. Novy \\ Reproductive Sciences, Oregon National Primate Research Center OB/GYN and Pediatrics, Oregon Health \\ Sciences University OB/GYN University of Washington
}

Objective: Intra-amniotic infection (IAI) is a common occurrence in pregnancy and is associated with preterm birth and other adverse neonatal sequelae. However, the early diagnosis of IAI is difficult and depends upon insensitive or non-specific tests. We followed an amniotic fluid (AF) proteomics-based analysis to identify peptides that could be used for sensitive and specific diagnostic tests for IAI in rhesus monkeys with experimental IAI and in humans.

Study design: Surface-enhanced laser desorption ionization (SELDI-TOF, Ciphergen Biosystems), one- and twodimensional gel electrophoresis, and tandem mass spectrometry (MS/MS) were used to characterize AF protein expression in 7 chronically instrumented pregnant rhesus monkeys before and after experimental IAI with group B streptococci'. Automated analysis of MS/MS spectra was performed with SEQUEST software (ThermoFinnigan) and de novo sequencing of all spectra with Lutefisk 1900 vI.2.5 software. AF protein profiles identified in experimental IAI were then tested in a cohort of 33 women with preterm delivery associated with subclinical IAI $(n=\mathrm{II})$, preterm delivery without IAI $(n=\mathrm{II})$, and preterm contractions with subsequent term delivery $(n=\mathrm{II})$.

Results: Detailed SELDI-TOF spectra revealed differential peptide expression between infected and non-infected primate $\mathrm{AF}$ in the $3-5 \mathrm{kD}$ and in the $10-12 \mathrm{kD}$ regions. An II kD peak was seen in all animals after infection and in no animal before infection. This II kD peak was observed $12 \mathrm{~h}$ after infection and preceded increases in uterine contractions or maternal signs and symptoms of IAI. In the human cohort, the II kD peak was also identified in II/II patients with subclinical IAI, in $2 / I I$ with preterm delivery without IAI, and in $0 / I I$ with preterm labor and term delivery without infection $(p<0.0 \mathrm{I})$. Protein identification within the II $\mathrm{kD}$ peak was obtained by in-gel trypsin digestion and MS/MS analysis. Proteins identified included azuricidin, calgranulin-B, S100 calcium-binding protein, and a unique fragment of insulin growth factor-binding peptide- I(IGF-BPI). Western blot analysis confirmed the differential expression of these peptides in the setting of infection.

Conclusion: This is the first observation of differentially expressed proteins within AF in the setting of IAI using proteomics-based analysis. The proteome profile described with experimental primate IAI was $100 \%$ sensitive and $91 \%$ specific in detecting subclinical IAI among a human cohort in preterm labor. We identified differentially expressed proteins that have not been previously associated with IAI but which have immunoregulatory properties. These proteins can potentially be used in the early detection of IAI.

Supported by NIHAI42490, HD06159

1. Gravett M. Am J Obstet Gynecol 1994;17:1660-7 


\title{
Impaired fertility in women with subclinical PID
}

\author{
H.C. Wiesenfeld, M.A. Krohn, S.L. Hillier, A.J. Amortegui and R.L. Sweet \\ Department of Obstetrics, Gynecology and Reproductive Sciences/Magee-Women's Research Institute, \\ University of Pittsburgh, $P A$
}

Objectives: Substantial retrospective data have accrued to date indicating that subclinical PID is an important cause of infertility. Previous studies have demonstrated that subclinical PID is present in $25 \%$ of women with chlamydial or gonorrhoeal infection and 15\% of women with bacterial vaginosis. We compared the rate of infertility among women with and without subclinical PID.

Study design: This was a prospective observational study of 767 women aged 15-30 years, at risk for PID. Subclinical PID was defined as the presence of histologic endometritis in the absence of signs or symptoms of acute PID. Participants were interviewed every 4 months concerning subsequent reproductive events. Sexually active women who did not conceive during I year of unreliable contraceptive use were categorized as infertile. Stepwise logistic regression was performed with variables associated with infertility on univariate analysis.

Results: Of the 767 participants, 224 became pregnant and I2I were infertile. Women with subclinical PID were at increased risk for infertility compared with women without subclinical PID.

Table I Factors associated with infertility

\begin{tabular}{lccc}
\hline Association & Infertile, $n=12 I$ & Fertile, $n=224$ & Adjusted RR, (95\% Cl) \\
\hline Subclinical PID & $17 \%$ & $10 \%$ & $2.1(1.1,4.1)$ \\
C. trachomatis infection & $20 \%$ & $21 \%$ & $0.7(0.3,1.6)$ \\
N. gonorrhoeae infection & $17 \%$ & $10 \%$ & $1.4(0.5,3.3)$ \\
Cigarette use & $70 \%$ & $59 \%$ & $1.7(1.1,2.8)$ \\
No previous pregnancies & $48 \%$ & $36 \%$ & $1.7(1.1,2.8)$ \\
\hline
\end{tabular}

Conclusion: Women with subclinical PID had a doubling of infertility compared with women without subclinical PID. These results represented the first prospective data confirming that subclinical PID is an important cause of infertility. Given the vast number of women with lower genital tract infections, these findings indicate that a substantial number of women of reproductive age are at risk for infertility.

Supported by NIH AI 41624 


\title{
Why nonoxynol-9 failed to prevent acquisition of Neisseria gonorrhoeae and to decrease vaginal lactobacilli in clinical trials
}

\author{
B.J. Moncla and S.L. Hillier \\ Department of Obstetrics and Gynecology and Magee-Women's Research Institute, University of Pittsburgh, \\ USA
}

Objective: Nonoxynol-9 $(\mathrm{N}-9)$ is a detergent-based topical contraceptive which has been evaluated as a topical microbicide for prevention of STDs/HIV. Published in vitro studies reported MIC's for N. gonorrhoeae (GC) and lactobacilli of $0.1-0.6 \%$. However, clinical trials of products containing $3-6 \% \mathrm{~N}-9$ have failed to demonstrate protection from GC and other STI's, or reduction of vaginal lactobacilli. The objective of this study was to test the hypothesis that $\mathrm{N}-9$ fails to protect against GC or destroy lactobacilli because the organisms are not sensitive at concentrations where they are inhibited using standard MIC assays.

Study design: Reference and clinical isolates of GC and lactobacilli were tested for MICs and minimum cidal concentration (MCC). MICs were performed by the standard NCCLS method, and MCCs were determined by evaluating viability after 30 -min exposures to $0.01-20 \% \mathrm{~N}-9$, followed by detection of growth after removing the test organisms from exposure to $\mathrm{N}-9$.

Results: MICs of $>5 \%$ for N-9 were observed for 18 of $26(69 \%)$ of GC isolates tested, all of which demonstrated MCC's of $>20 \%$. The 8 remaining isolates had MICs and MCCs of $<1.0 \%$. The GC strain ATCC 19425, which was used in earlier in vitro testing studies, was sensitive to N-9 in these studies, but the GC strain ATCC 49226 was not. On MCC assays $8 \mathrm{~L}$. crispatus $\left(\mathrm{H}_{2} \mathrm{O}_{2}+\right)$ isolates were resistant to $\mathrm{N}-9$, but sensitive to $\mathrm{N}-9$ on MICs $(<0.5-2 \%)$. Of $7 \mathrm{~L}$. iners $\left(\mathrm{H}_{2} \mathrm{O}_{2}-\right)$ strains, all were resistant to $\mathrm{N}-9$ with MCCs of $4-20 \%$, but were sensitive on standard MIC assays $(<0.1-2 \%)$.

Conclusion: Most isolates of GC (69\%) and lactobacilli (100\%) were resistant to N-9 at concentrations attainable in vivo with any of the formulations of $\mathrm{N}-9$. Earlier reports relied on reference strains sensitive to $\mathrm{N}-9$ and included too few clinical isolates to determine the true prevalence of resistance. Standard MIC assays do not have a strong correlation with cidal activity, nor do they correlate well with the results of clinical trials of $\mathrm{N}-9$. 


\title{
High prevalence of clindamycin-resistant anaerobic Gram-negative rods after treatment with intravaginal clindamycin for bacterial vaginosis
}

\author{
R.H. Beigi, M.N. Austin, I.S. Macio, L.A. Meyn and S.L. Hillier \\ University of Pittsburgh and Magee-Women's Research Institute, Pittsburgh, USA
}

Objectives: To determine whether detectable increases in MICs exist above the cut-off point for resistance to clindamycin and metronidazole in anaerobic bacteria after intravaginal treatment for bacterial vaginosis (BV). Study design: A randomized open-label clinical trial of treatment for BV comparing vaginal metronidazole with vaginal clindamycin was conducted among 119 non-pregnant women aged 18-45 years. After treatment the participants had three follow-up visits, at 7-12 days, 35-45 days and 70-90 days. At all visits quantitative vaginal cultures were performed and predominant anaerobic isolates underwent antimicrobial susceptibility testing for metronidazole and clindamycin resistance by agar dilution methods.

Results: Of 69 women with susceptibility data available for analysis, 33 were randomized to metronidazole and 36 to clindamycin. We detected marked and sustained increases in the number of women having anaerobic Gram-negative rods with resistance to clindamycin among women receiving clindamycin but not among those receiving metronidazole (Figure I).

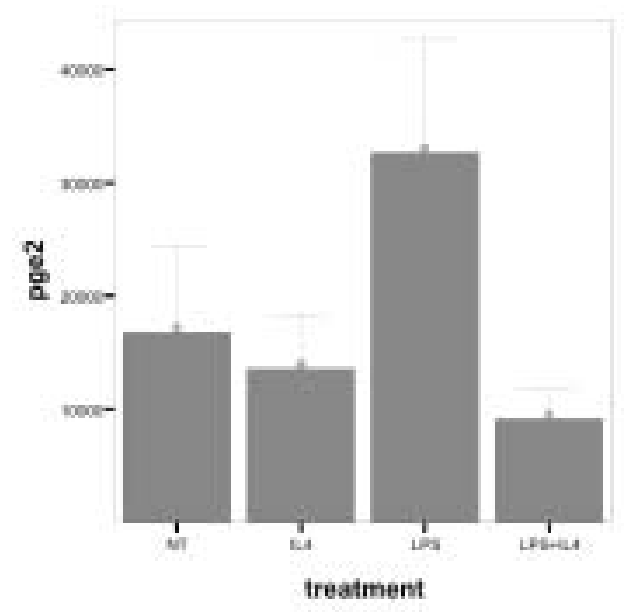

In contrast, only two anaerobic isolates had resistance to metronidazole, one at baseline and one at the first follow-up visit. There was no increase in the number of metronidazole-resistant isolates in women treated with metronidazole.

Conclusion: Use of intravaginal clindamycin for the treatment of $\mathrm{BV}$ is associated with a significant increase in the prevalence of clindamycin-resistant anaerobic bacteria in the vagina. This finding may have important clinical implications considering the vast number of bacteria in the vagina exposed to clindamycin.

Supported by an unrestricted research grant from 3M Pharmaceuticals. 


\title{
Assessment of the bacteriocinogenic potential of vaginal streptococci and enterococci against vaginal lactobacilli
}

\author{
D.C. DeZwaan and V. Pybus
}

Objectives: The vaginal syndrome bacterial vaginosis (BV) is characterized by an alteration in the vaginal microflora such that lactobacillus populations that are generally dominant in healthy women decline in concentration and are replaced by Gardnerella vaginalis, obligate anaerobes and genital mycoplasmas. At present there is a lack of ecologically based explanations, in particular of microbial interactions, to account for the decline in lactobacillus populations potentially leading to the establishment of a BV-associated microflora. The production of bacteriocins antagonistic to the growth of vaginal lactobacilli is a possible mechanism which could account for this. Since streptococci and enterococci of non-vaginal origin have been well-documented for their ability to produce bacteriocins, the aim of this study was to assess the bacteriocinogenic potential of vaginally-derived isolates of these bacteria against the growth of vaginal lactobacilli.

Study design: Using the deferred antagonism agar plate technique, 123 vaginal streptococci/enterococci specimens were tested for the production of bacteriocin-like inhibitors against 16 strains of vaginal lactobacilli. Results: Of the 123 isolates tested, 28 inhibited the growth of one or more vaginal lactobacillus isolates. Based on phenotypic characterization assays, the 123 strains were separated into four categories: enterococci, streptococci, group D streptococci and other, with 9, 59, 45 and 10 isolates in each category, respectively. The percentage of strains inhibiting the growth of one or more lactobacillus strains was $0 \%$ streptococci, $33 \%$ enterococci, $33 \%$ group D streptococci, and $100 \%$ of others.

Conclusion: Almost one quarter of the 123 strains of vaginal streptococci/enterococci tested were antagonistic to the growth at least $I$ of the 16 strains of vaginal lactobacilli. Future studies will further assess the in vivo significance of these anti-lactobacillus effects in both the establishment and the recurrence of BV. 


\title{
Results, potential consequences and the situation after discontinuation of the Thuringia Prematurity Preventional Campaign 2000
}

\author{
U.B. Hoyme ${ }^{1}$, J. Hübner ${ }^{2}$, U. Möller ${ }^{3}$ and E. Saling ${ }^{4}$ \\ ${ }^{1}$ Department of Obstetrics and Gynaecology, Helios Klinikum Erfurt, Germany, ${ }^{2}$ Department of Obstetrics and \\ Gynaecology, Helios Klinikum Aue, Germany, ${ }^{3}$ Department of Obstetrics and Gynaecology, Friedrich-Schiller- \\ University, Jena, Germany and ${ }^{4}$ Institute of Perinatal Medicine, Berlin, Germany
}

Objective: Bacterial vaginosis and other infectious conditions are major causes of late miscarriage and early premature birth. We aimed to investigate prospectively the effectiveness of a program for prematurity prevention.

Study design: In part A, pregnant women in Erfurt were asked to perform self-measurements of vaginal $\mathrm{pH}$. The women were instructed to see their physician if abnormal values $(\mathrm{pH} \geqslant 4.7)$ or other risk factors were present, in order to get these confirmed and to start lactobacillus therapy or, in the case of bacterial vaginosis, to treat with clindamycin intravaginal cream. In part B, in March 2000 a similar pH-screening program was initiated in order to reduce prematurity in the State of Thuringia. According to the study design, a significant decrease of prematurity was hypothetically expected for the second half of the year 2000.

Results: In part A, 73 of 381 women were identified as risk cases. In this study, the prematurity rate was $8.1 \%$ in the self-measurement/intervention group vs $12.3 \%$ in the control group $(p<0.05, n=234$ ) ; $0.3 \%$ vs $3.3 \%$ of the neonates were premature with a gestational age of $<32$ weeks $(p<0.01)$. PROM was registered in $22.8 \%$ vs $30.8 \%(p<0.001)$, respectively. In part $B$, an overall decrease of prematurity from $7.68 \%$ to $6.81 \%$ and a reduction of cases at $<32$ weeks from $3.22 \%$ to $2.39 \%$ was observed $(n=1600)$; premature rupture of the membranes was seen in 19 vs 0 pregnancies with early prematurity. Data from 16276 women are available for the State: a significant reduction of early prematurity from I.58\% to $0.99 \%$, respectively, was seen $(p<0.00 \mathrm{I})$. Conclusion: Comparing low birthweights, a significant reduction of cases was achieved in all groups. On the basis of these data we recommend the extension of the campaign throughout the whole of Germany. In 200I/2002, after discontinuation of the campaign, the prematurity rate (i e. neonates $<1000 \mathrm{~g}$ ) increased, further supporting this conclusion. 


\title{
Oral presentations: session V
}

\section{The feasibility and effect of HSV serotesting of partners of HSV-2 sero-negative pregnant women}

\author{
C. Gardella, Z. Brown, A. Akmal, S. Berry, S. Selke, L. Corey and A. Wald \\ Departments of Obstetrics, Gynecology, Medicine and Epidemiology, University of Washington,
}

Objectives: To determine the acceptability of HSV serotesting of partners among susceptible pregnant women and compliance with safer-sex counseling during pregnancy.

Study design: HSV-2 sero-negative women receiving prenatal care at the University of Washington were invited to participate in this study at between 16 and 28 weeks' gestational age. Participants were asked to invite their partners for HSV testing to identify HSV serodiscordance, and to keep diaries of sexual activity during pregnancy. Standardized abstinence and safer-sex counseling was given based on risk status. Enrollment is ongoing.

Results: Between July 2001 and April 2003, 23I women were invited to participate and 99 were enrolled. Compared with participants, women who declined were significantly older (median 32 vs 29 years of age, $p=0.002$ ) but of similar ethnicity (white $82 \%$ vs other $72 \%, p=0.08$ ) and insurance status (private $78 \%$ vs $77 \%$, $p=0.4)$. The partners of $84(85 \%)$ women were tested, and of these couples $69(82 \%)$ had concordant HSV serologies or results that placed the male partner at risk; 13 (15\%) had discordant HSV serologies that placed the woman at risk for HSV-I; I (I\%) had serologies that placed the woman at risk for HSV-2 and -I; and I (I\%) had serologies that placed the woman at risk for both HSV-I and HSV-2.

Table I Features of sexual relationship and results of serotesting

\begin{tabular}{|c|c|c|c|c|}
\hline Feature & $\begin{array}{l}\text { Partner not tested } \\
\qquad(n=15)\end{array}$ & $\begin{array}{c}\text { At risk for HSV-I or } \\
\text { HSV-2 }(n=15)\end{array}$ & $\begin{array}{l}\text { No risk } \\
(n=69)\end{array}$ & $p$ Value \\
\hline Number of partners during pregnancy & I (range I-3) & I (range I-I) & I (range I-I) & $<0.001$ \\
\hline Duration of partnership (months) & $5 \mathrm{I}$ (range $\mathrm{I}-2 \mathrm{I} 3)$ & 69 (range 12-184) & 6I (range 9-247) & 0.17 \\
\hline Frequency receptive oral sex per week & 0 (range $0-0.4)$ & 0 (range $0-1)$ & 0 (range $0-2$ ) & 0.54 \\
\hline Frequency genital sex per week & 0.3 (range $0-3$ ) & 0.6 (range $0-5$ ) & 0.6 (range $0-3$ ) & 0.9 \\
\hline Unprotected receptive oral sex & $5 / 15(33 \%)$ & $2 / 15(13 \%)$ & $19 / 69(28 \%)$ & 0.42 \\
\hline Unprotected genital sex & $9 / 15$ (60\%) & $9 / 15(60 \%)$ & $53 / 69(77 \%)$ & 0.35 \\
\hline$\%$ Receptive oral sex acts without dam & $100 \%$ & $100 \%$ & $100 \%$ & 1.0 \\
\hline$\%$ Genital sex acts without condom & $100 \%$ & $100 \%$ & $100 \%$ & 0.85 \\
\hline
\end{tabular}

Conclusion: HSV serotesting of partners is feasible. Preliminary data suggest that compliance with abstinence and safer sex is poor in pregnancy, even among women known to be at risk for HSV acquisition by partner testing. This suggests that other strategies to prevent HSV acquisition during pregnancy are needed.

Supported by NIAID grant AI-30731 


\title{
Prevention of chlamydia and gonorrhea: results of a 24-month randomized trial of behavioral intervention in minority women
}

\author{
R.N. Shain, J.M. Piper, A.E.C. Holden, J.D. Champion, S.T. Perdue, J.E. Korte and \\ F. Guerra
}

Objectives: Our aims were firstly to confirm the results of a previous triall showing that our standard cultureand gender-specific behavioral intervention helped reduce rates of gonorrhea and chlamydia for 12 months; secondly, to extend evaluation to 24 months; and thirdly, to test the efficacy of an enhanced intervention arm, i.e. standard intervention plus the option of support groups.

Study design: Mexican-American and African-American women ( $90 \%$ under 30 years of age) with a current nonviral STD were interviewed, counseled, examined and randomized to standard intervention, enhanced intervention, or controls. Participants were interviewed and screened at 6, 12 and 24 months, and were given a brief interview and option of examination at 18 months. Women were encouraged to return to the clinic for suspected problems. Multiple logistic regression analysis (intention to treat), controlling for group differences in variables independently related to outcome, was used to determine group differences in follow-up infection rates.

Results: Over 800 women were enrolled; retention rates at 12 and 24 months were over $90 \%$; the intervention show rate was $96 \%$; and $39 \%$ of those in enhanced intervention opted to attend support groups. The analysis sample at 0-24 months comprised 693 participants. Adjusted cumulative 24-month infection rates were 38.7\% (controls), $27.0 \%$ (standard intervention) and $23.6 \%$ (enhanced intervention). When enhanced-intervention participants were divided according to attendees and non-attendees, adjusted rates were $19.0 \%$ and $26.1 \%$, respectively. Corresponding adjusted odds ratios and confidence limits, compared with controls, were:

standard intervention: $0.58(0.37-0.91)$

enhanced intervention (overall): $0.49(0.32-0.76)$

attendees only: $0.37(0.20-0.70)$

non-attendees only: $0.56(0.35-0.91)$.

Conclusion: This study confirms the efficacy of our standard intervention and demonstrates efficacy of both interventions for at least 24 months. Enhanced-intervention participants had slightly lower infection rates than standard-intervention participants, but this effect was seen only among those who actually attended one or more group meetings.

Shain et al. N Eng J Med1999; 340:93

$180 ・$ INFECTIOUS DISEASES IN OBSTETRICS AND GYNECOLOGY 


\title{
Rapid detection of group B streptococcus and Escherichia coli in amniotic fluid using real-time fluorescent PCR
}

\author{
M. Straka, O. Johnson, C. Blackmon, S. Stassen, D. Streitman and D. Stamilio
}

Objectives: To establish reliability and validity of real-time fluorescent polymerase chain reaction (PCR) for early detection of bacterial invasion of the amniotic cavity.

Study design: Amniotic fluid samples from 40 patients undergoing mid-trimester genetic amniocentesis were incubated for 6 hours at $37^{\circ} \mathrm{C}$, cultured on media specific for GBS and E. coli, and analyzed by conventional PCR and real-time fluorescent PCR. Samples were re-analyzed for study pathogen DNA after inoculation with $10^{4} \mathrm{cfu} /$ $\mathrm{ml}$. Pathogen DNA was extracted and purified through two precipitations. DNA primers designed for unique target sequences of GBS and E. coli were used in real-time fluorescent PCR. The lower limit of detection for each pathogen was established using logarithmic inoculants of negative amniotic fluid samples. Negative samples were inoculated with 25 non-GBS, non- $E$. coli bacterial species at $10^{4} \mathrm{cfu} / \mathrm{ml}$ and re-analyzed with real-time fluorescent PCR.

Results: All samples were negative for evidence of GBS and E. coli. All inoculated samples produced strong fluorescent signal with real-time PCR which failed to detect the presence of non-GBS, non-E. coli bacterial species. Average total sample processing time was $7 \mathrm{~h} 40 \mathrm{~min}$.

Conclusion: Real-time fluorescent PCR is a valid and reliable method for detection of specific pathogens in amniotic fluid. This technique is sensitive to low inoculation levels and has potential in clinical management as a rapid, reliable detection method for GBS and $E$. coli in intrauterine infection. 


\title{
Evaluation of real-time polymerase chain reaction assays for detection and confirmation of Neisseria gonorrhoeae in clinical samples
}

\author{
S.M. Garland ${ }^{1}$, S.N. Tabrizi ${ }^{1}$, S. Chen ${ }^{1}$, M.A. Cohenford ${ }^{2}$, B.B. Lentrichia ${ }^{2}$, \\ E. Coffman ${ }^{2}$, T. Shultz ${ }^{3}$ and J.W. Tapsall ${ }^{3}$ \\ ${ }^{1}$ Molecular Microbiology Laboratory, The Royal Women's Hospital, Victoria, Australia, ${ }^{2}$ CYTYC \\ Corporation, Boxborough, MA, USA and ${ }^{3}$ Department of Microbiology, Prince of Wales Hospital, Randwick, \\ Australia
}

Objectives: To develop real-time confirmatory PCR assays for detection of $N$. gonorrhoeae in clinical samples testing positive by Roche COBAS Amplicor.

Study design: Assay specificity was evaluated using strains of 40 Neisseria species, 120 ATCC and clinical strains of $N$. gonorrhoeae and other bacteria. In addition, serial dilution of $N$. gonorrhoeae strain was used to assess sensitivity of the assays. In all, 124 positive $N$. gonorrhoeae specimens were selected from specimens of more than 3000 women tested previously in various studies in Australia and the Pacific Islands, based on their positivity by Roche COBAS Amplicor. A further 50 samples negative by COBAS Amplicor were also randomly selected.

Results: Related Neisseria species were negative by both assays, whereas 120 clinical isolates of $N$. gonorrhoeae were all positive. Both assays generated a sensitivity of I copy per reaction. Overall 73 of 122 COBAS-positive samples were confirmed as positive $(59.8 \%)$. The confirmation rate was found to be directly proportional to the optical density of the COBAS PCR, with highest confirmation of $93.5 \%$ obtained with samples having an OD of higher 3.5. The $16 \mathrm{~S}$ and LCx assays produced similar results to the real-time assays, indicating similar sensitivities and specificities to the FRET and MB probe assays.

Conclusion: Data from this study highlight the importance of a confirmatory step for diagnosis of $N$. gonorrhoeae by COBAS PCR. It is recommended that confirmation be part of the diagnostic algorithm of all diagnostic laboratories performing this assay. 


\title{
Oral presentation. Research on preterm birth: where should we be going?
}

\section{Complexity-informed approaches to prevention of low birth weight and short gestation}

\author{
J.A. McGregor, J.I. French and C. Hobel \\ Cedars-Sinai Medical Center/UCLA, Los Angeles, CA, USA Obstetrix, Tucson, AZ, USA
}

Background: Low birth weight (LBW) and short gestation (SG) are complex diseases mediated by interactions between maternal and embryonic, fetal, trophoblastic genomes and proteomes interacting with multiple environmental factors. These factors include infection, inflammation and other processes.

Objective: We aimed to review breakthroughs in knowledge in diverse fields that might inform and prompt new approaches to research into and clinical management of LBW/SG.

Study design: We systematically reviewed and analyzed advances in diverse fields, including advances in decision analysis, microbiology, genomics, proteonomics, infectious diseases and reproductive biology. Resources included computerized database searches, extensive reviews (Science, I April 2002), and consultations with experts (Plexus Institute, Entelos).

Results: LBW/SG is now understood to be in part caused by potentially preventable infections and inflammatory processes. LBW/SG is considered a supercomplex disorder: processes involve both maternal and embryonic/fetal and trophoblastic tissues. Complex diseases are characterized by:

multiple causes

inter-relatedness of causes

non-linear responsiveness

time-influenced processes

stochastic resonance

responsiveness to comprehensive treatment approaches.

Examples of complexity in LBW/SG include synergism between lower reproductive tract infection(s), cervical factors, and gestational bleeding in genetically predisposed hosts. Recognition of complexity mandates research into comprehensive approaches to individually determined, interacting and time-linked etiologic factors. Application of complexity has led to the "inducer, enhancer, trigger models" in the treatment of asthma.

Conclusion: Acknowledgment of complexity prompts re-analysis of investigational and clinical approaches to prevention of LBW/SG. Recognition of multiple, inter-related causations, non-linear responsiveness, and the need for comprehensive "inducer, enhancer, trigger" models based genomically in susceptible individuals, offers rich and novel opportunities for the prevention of LBW/SG. 


\title{
Research by committee: why maternal-fetal network trials failed to prevent prematurity/low birthweight caused by bacterial vaginosis and trichomoniasis
}

\author{
J.A. McGregor and J.I. French \\ Cedars-Sinai Medical Center/UCLA, Los Angeles, CA, USA Obstetrix, Tucson, AZ, USA
}

Background: Research design strongly influences outcomes in large multicenter controlled trials.

Objective: We aimed to analyze design-derived sources of bias, error and misinterpretation for publicly funded maternal-fetal network (MFN) trials evaluating treatment of bacterial vaginosis (BV) and trichomoniasis (TV) in pregnancy.

Study design: Abstracts, presentations, final publications and published comments regarding MFN trials focusing on treatment of BV and TV in pregnancy were reviewed and analyzed.

Results: Analyses of both trials demonstrated concerns regarding both internal and external validity of study design, performance, analyses, interpretation and promulgated conclusion. These concerns focus on:

discrepancies regarding major study design features

inappropriate non-evidence-based treatment selections and use of non-CDC-P recommended study treatments non-biologically-based study hypotheses

biologically significant delays in study treatments

use of excessive and possibly toxic dosage regimens

inappropriate systematic case exclusion

disregard of fetal immune ontogeny and development

inappropriate interpretation.

Conclusion: Design and analytic features of these MFN trials prompt important concerns regarding internal and external validity of these publicly funded studies. Design and selection of future MFN studies should involve competitive processes and be peer reviewed. 


\title{
Scientific poster session. Poster \# I
}

\section{Transplacental passage of vancomycin in the ex vivo human perfusion model}

\author{
M. Hnat, J. Gainer, G.D. Wendel Jr and R.E. Bawdon
}

Objective: To determine maternal-fetal transplacental passage of vancomycin in the ex vivo human placental perfusion model.

Study design: In all 6 term placentas were collected immediately after delivery and perfused with physiologic medium using the single cotyledon perfusion system. Trough and peak vancomycin concentrations (range: 7.7$16.4 \mathrm{~g} / \mathrm{ml}$ and $31.4-51.5 \mathrm{~g} / \mathrm{ml}$, respectively) were added to the maternal medium and perfused through the maternal circulation of the cotyledon. Over a I-h period in an open system, samples of the perfusate were collected at defined intervals from the fetal venous catheter and from the maternal effluence to assess vancomycin transfer. Thereafter, the system was closed for $\mathrm{I}-5 \mathrm{~h}$ to establish accumulation. Transport fraction and clearance indexes were calculated by perfusing antipyrine $\mathrm{C}^{14}$ (positive control). High-pressure liquid chromatography determined vancomycin and antipyrine concentrations.

Results: Mean vancomycin maternal peak and trough concentrations and mean peak and trough clearance indexes are listed in Table I. For each placenta, transport fraction for antipyrine was $>1.85$ with a single pass of $>40 \%$. No accumulation of vancomycin was noted when the system was closed for $\mathrm{I}-5 \mathrm{~h}$. The mean peak clearance index was zero after perfusing the placenta for up to $5 \mathrm{~h}$ with $35.8 \mathrm{~g} / \mathrm{ml}$ of vancomycin.

Conclusions: Transplacental passage of vancomycin was minimal in the ex vivo human placental perfusion model, with no detectable accumulation.

Table I Mean vancomycin concentrations and clearance indexes

\begin{tabular}{lcccc}
\hline & \multicolumn{2}{c}{ Maternal concentration $(\mu \mathrm{g} / \mathrm{ml})$} & \multicolumn{2}{c}{ Clearance index } \\
Placenta \# & Trough & Peak & Trough & 0.035 \\
\hline & - & 51.5 & - & 0.000 \\
2 & 16.4 & 33.0 & 0.00 & 0.080 \\
3 & 11.7 & 31.4 & 0.17 & 0.014 \\
4 & 7.7 & 40.1 & 0.05 & - \\
5 & 14.8 & - & 0.00 & 0.000 \\
6
\end{tabular}




\title{
Scientific poster session. Poster \#2
}

\section{Improving universal screening for HIV in pregnancy}

\author{
B.L. Anderson, H.N. Simhan and D.V. Landers \\ Department of Obstetrics, Gynecology and Reproductive Sciences, University of Pittsburgh, PA
}

Objective: To evaluate the effect of implementation of an HIV screening educational intervention on universal screening for HIV in pregnant patients in an ambulatory clinic setting.

Study design: In this retrospective cohort study, frequencies of offering and of acceptance of HIV testing was compared before and after hiring an HIV-focused nurse to draw awareness to HIV screening and management. The nurse educated prenatal care providers to facilitate counseling and encourage acceptance of HIV screening among pregnant women. The records of 293 women seeking prenatal care before the educational intervention and of 206 women seeking prenatal care after the educational intervention were reviewed for offering and acceptance of HIV testing. Fisher's exact test and logistic regression were used to evaluate the relationship between the educational intervention and the offering and acceptance of HIV testing.

Results: The frequencies of offering and acceptance of HIV testing at first visit before the educational intervention were $96.5 \%$ and $74.8 \%$, respectively, and after the intervention were $99.5 \%$ and $84.3 \%$, respectively. These improvements were significant (offering, $\mathrm{OR}=7.27,95 \% \mathrm{Cl}=1.02-316.9$; acceptance, OR $=1.82,95 \% \mathrm{Cl}=1.14-2.88$ ). Women with a history of intravenous drug abuse, STD, or sex with a man who had been in jail were more likely to accept the test. Test acceptance was statistically significantly improved in the post-intervention group even after controlling for confounding variables with a forward stepping logistic regression model $(\mathrm{OR}=2.02,95 \% \mathrm{Cl}=1.2-3.39)$.

Conclusion: The addition of an HIV-focused nurse to the staff of an ambulatory clinic improved the frequency of test offering at first visit and acceptance of HIV testing in pregnant patients. 


\title{
Scientific poster session. Poster \#3
}

\section{Human papillomavirus infection and in vitro fertilization outcome}

\author{
A.M. Bongiovanni, S.D. Spandorfer, L.R. Paraskevas and S.S. Witkin \\ Weill Medical College of Cornell University, New York, USA
}

Objective: Human papillomavirus infection (HPV) causes a common infection of the female genital tract. The relationship between a cervical HPV infection and in vitro fertilization (IVF) outcome was investigated.

Study design: Cervical swabs collected from 84 consecutive women undergoing IVF were tested for HPV by a commercial assay (Digene). IVF parameters and outcomes were subsequently obtained and related to HPV status. Categorical chi-square testing and Student's $t$-test were used where appropriate. Pregnancy was defined as a rise in $\beta$-hCG, implantation rate as the number of embryos implanted/number of embryos returned, and clinical pregnancy as the presence of a fetal heartbeat.

Results: Of 13 (I5.5\%) women testing positive for HPV, 7 were high-risk, I was low-risk, and the remaining 5 were combined high-/low-risk HPV types. HPV status was unrelated to age, oocyte yield, fertilization percentage or number of embryos transferred. However, pregnancy, implantation and clinical pregnancy rates were strongly negatively associated with detection of HPV (Table I).

Table I Relation between HPV detection and IVF outcome

\begin{tabular}{lccc}
\hline Parameter & $H P V \pm(n=13)$ & $H P V-(n=7 I)$ & $p$ Value \\
\hline Age (years) & $37.0(5.1)$ & $36.5(5.1)$ & NS \\
Embryos transferred $(n)$ & $2.9(1.7)$ & $3.0(1.5)$ & NS \\
Pregnancy rate & $15.4 \%$ & $59.2 \%$ & 0.0037 \\
Implantation rate & $9.1 \%$ & $33.1 \%$ & 0.0166 \\
Clinical pregnancy rate & $15.4 \%$ & $53.5 \%$ & 0.0114
\end{tabular}

Conclusions: It is unclear whether cervical HPV directly inhibits IVF pregnancy or is a marker for another event. Since these women were in monogamous relationships, they probably became infected a long time previously. Their failure to spontaneously clear HPV suggested that they had diminished pro-inflammatory immunity. This might have reduced the success rate of implantation and could account for the observations. 


\title{
Comparison of DNA hyrbridization with Gram stain for the detection of bacterial vaginosis among asymptomatic pregnant women
}

\author{
K.A. Boggess ${ }^{1,2}$, P. Madianos ${ }^{2}$, A. Murtha ${ }^{2}$, S. Lieff, S. Hillier ${ }^{3}$, L. Rabe ${ }^{3}$ and S. \\ Offenbacher ${ }^{2}$ \\ ${ }^{1}$ Department of Obstetrics and Gyneccology, UNC, Chapel Hill, ${ }^{2}$ Center for Oral and Systemic Diseases, \\ UNC, Chapel Hill and ${ }^{3}$ Department of Obstetrics and Gynecology, McGee Women's
}

Objective: Bacterial vaginosis (BV) affects 10-15\% of pregnant women and has been linked with preterm birth and maternal infectious morbidity. Nugent created criteria for the diagnosis of BV based on relative proportions of reliable morphotypes identified by Gram stain. Checkerboard DNA hybridization identifies the presence of microbial DNA by using hybridizing labeled probes. Our objective was to compare DNA hybridization with Gram stain for the diagnosis of BV in asymptomatic pregnant women.

Study design: As part of the Oral Conditions and Pregnancy Study, vaginal specimens were collected at $<26$ weeks of gestation from 1115 women and stored at $-80^{\circ} \mathrm{C}$ until analysis. A random subset of 246 specimens was analyzed by Gram stain using Nugent criteria and by DNA hybridization. DNA hybridization was performed using the checkerboard method with probes developed to detect Lactobacillus spp., Gardnerella vaginalis, Prevotella bivia, Bacteroides ureolyticum and Mobiluncus curtisii. Any positive DNA result was compared with the Gram stain result using McNemar paired test and Spearman's correlation. Statistical significance was set at $p<0.05$.

Results: Of 246 specimens analyzed, I 44 (59\%) were negative for BV by Gram stain, 67 (27\%) were positive, and 35 (14\%) were scored as intermediate. DNA results were available for 237 specimens: 106 (45\%) were negative for any pathogen and I 3 I (55\%) were positive for one or more vaginal pathogens (76 were positive for one pathogen, 45 were positive for 2 pathogens and 10 for 3 or more pathogens). In all 197 paired Gram stain/ DNA results were available for analysis. DNA and Gram stain results were significantly correlated $(p<0.000 \mathrm{I})$. The sensitivity of DNA hybridization to detect BV was $93.5 \%$ (Table I).

Table I Sensitivity of DNA hybridisation compared with Gram stain

\begin{tabular}{lccc}
\hline DNA result & Gram: BV positive & Gram: BV negative & Total \\
\hline DNA positive & 58 & 48 & 106 \\
DNA negative & 4 & 87 & 91 \\
Total & 62 & 135 & 197 \\
\hline
\end{tabular}

The vast majority of specimens (216/236) were positive for Lactobacillus spp. by hybridization, but $7 \mathrm{I}$ of these were read as $0-2$ for Lactobacilusi spp. by Gram stain. Among the 35 intermediate Gram stains, 23 were positive for vaginal pathogens by DNA, and 30 were positive for Lactobacillus spp.

Conclusion: DNA hybridization is an effective method for detection of BV during pregnancy and may be a useful technique to study the biology of this common infection during pregnancy.

Supported by NIDCR R01-DE-12453 and HD K12 01441.

I88 - INFECTIOUS DISEASES IN OBSTETRICS AND GYNECOLOGY 


\title{
HIV-I RNA levels in the female genital tract differ according to collection method: analysis of cervicovaginal lavage and sno- strip collections
}

\author{
S. Cu-Uvin, B. Snyder, S. Donahue, J. Allega, J. Hogan, J. Harwell, J. Kurpewski, \\ C.C.J. Carpenter, T. Flanigan, K. Mayer and A. Caliendo
}

Objective: There is no current consensus on the best method to collect female genital secretions for measurement of HIV shedding. We aimed to compare cervicovaginal lavage (CVL) with sno-strip collections for measuring genital tract HIV-I RNA.

Study design: To assess the effect of antiretroviral therapy on HIV in the genital tract, 78 paired CVL and snostrip samples were collected from a cohort of women enrolled in a longitudinal study. Three sno-strips were used as wicks to collect primarily cell-free virions from the endocervical canal. Each sno-strip adsorbs approximately $8 \mathrm{I}$ of specimen and adsorption usually takes I min. CVL was performed by aiming a continuous stream of $10 \mathrm{ml}$ of normal saline directly at and into the cervical os to bathe the endocervix and ectocervix. The fluid was allowed to pool in the posterior fornix and then aspirated. Plasma viral load (PVL) was also measured. HIV-I RNA levels were quantified by NASBA with a lower detection limit of 400 copies $/ \mathrm{ml}$. An exact version of the McNemar's test was used in the statistical analysis.

Results: We found that $65 \%$ of the women had PVL $>400$ copies $/ \mathrm{ml}$ (range: $<400-890000$ copies $/ \mathrm{ml}$ ), 26\% had CVL HIV-I RNA $>400$ copies/ml (range: $<400-480000$ copies $/ \mathrm{ml}$ ), and $40 \%$ had sno-trip HIV-I RNA $>400$ copies/ml (range: $<400-2160000$ copies/ml). CVL HIV-I RNA was greater than PVL in 5.7\% of women, and sno-trip HIV-I RNA was greater than PVL in $40.4 \%$ of women $(p<0.000 \mathrm{I})$. Among women with PVL $<400$ copies $/ \mathrm{ml}, 92.6 \%$ and $96.3 \%$ had CVL viral load and sno-strip viral load below detectable limits, respectively $(p=$ I.000). Among women with PVL $>400$ copies $/ \mathrm{ml}, 35 \%$ and $58.8 \%$, respectively, had CVL viral load and sno-strip viral load $>400$ copies $/ \mathrm{ml}(p=0.0042)$.

Conclusions: Among women with detectable PVL, the proportion of detectable sno-strip viral load was significantly greater than the proportion of detectable CVL viral load. The sno-strip collection method detected more HIV-I RNA in genital secretions. These findings may in part be due to the dilution effect of CVL or to the difference in subcompartments sampled. 


\title{
Effect of daily directly observed therapy on genital tract and plasma HIV-I RNA in HIV-infected, substance-abusing women: pilot study
}

\author{
S. Cu-Uvin, J. Mitty, C.C.J. Carpenter, A. Caliendo, M. Stenzel, S. Donahue, \\ J. Allega, M. Mckenzie, G. Macalino, S. Chapman and T. Flanigan
}

Objective: To determine if daily directly observed therapy (DOT) using a once-daily combination antiretroviral regimen for 6 months, followed by less intensive DOT, is feasible and may result in effective viral suppression in both genital tract and blood plasma of substance-abusing women.

Study design: A cohort of 12 HIV-positive women with active substance abuse during the preceding 90 days, previous antiretroviral treatment and history of non-adherence was enrolled. Physician evaluation had suggested that the subjects would respond to a once-daily regimen of $d 4 t$, ddl, 3TC, and either nevirapine or efavirenz or ritonavir and saquinavir. A "near-peer" outreach worker met the participants daily to deliver medications and observe participants take the dose. Plasma and cervicovaginal lavage (CVL) HIV-I RNA were quantified by NASBA. The lower limit of detection was 400 copies $/ \mathrm{ml}$.

Results: AIDS-related complications caused the death of I woman; I woman with continued virologic failure was disenrolled as soon as genotyping revealed resistance to DOT medications; 3 women did not follow up with study visits; 6 of the remaining 7 women had at least 6 months' data on plasma and CVL viral load available. At

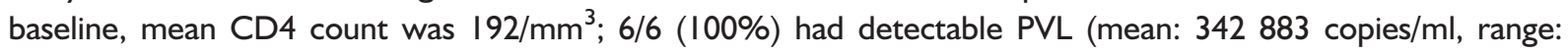
2300-I 200 000); and 5/6 (83\%) had detectable CVL HIV-IRNA (mean : 17767 copies/ml, range: <400-47 000). At I month, 3/6 (50\%) and 5/6 (83\%) had plasma and CVL HIV-I RNA $<400$ copies/ml, respectively. At 3 months, 5/6 (83\%) and 6/6 (I00\%) had plasma and CVL HIV-I RNA <400 copies/ml, respectively. At 6 months, mean CD4 count was $268 / \mathrm{mm}^{3}$ and $6 / 6$ (100\%) women had plasma and cervicovaginal viral load below detectable limits. Mean log drop in plasma viral load was $2.9 \log _{10}$ and $1.6 \log _{10}$ for CVL viral load. Mean increase in CD4 count was $76 / \mathrm{mm}^{3}$.

Conclusion: Strategies of DOT are effective in suppressing plasma and genital tract viral load among substanceabusing women. This may have an impact on sexual as well as vertical transmission of HIV. 


\title{
Scientific poster session. Poster \#7
}

\section{Improving appropriate use of antifungal medications: the role of an over-the-counter vaginal $\mathrm{pH}$ self-test device}

\author{
S. Roy, J.C. Caillouette, J.S. Faden, T. Roy and D.E. Ramos
}

Objective: We aimed to determine whether women can understand and use the vaginal $\mathrm{pH}$ device in the diagnosis of vaginitis;' to compare the vaginal $\mathrm{pH}$ readings determined by subjects and by healthcare providers; and to determine whether vaginal $\mathrm{pH}$ can reduce inappropriate over-the-counter (OTC) antifungal medication use and improve the correct diagnosis of vaginitis ${ }^{2}$.

Study design: In all, I5I women indicated their beliefs about the cause of their vaginal infection, read the instructions of the vaginal $\mathrm{pH}$ device package insert, used the device and interpreted the findings. The subjects' interpretations were compared with results obtained by healthcare providers who were blinded to subject findings.

Results: The women $(<96 \%)$ stated that they could easily read the instructions, use the vaginal $\mathrm{pH}$ device and interpret the readings. They obtained the same readings as healthcare professionals (kappa $=0.9$ ). Restricting the use of OTC antifungal medications to those individuals with vaginitis symptoms and vaginal $\mathrm{pH} \leqslant 4.5$ significantly reduced inappropriate use by approximately $50 \%$ (Fisher's exact test, $p=0.018$ ). Conversely, seeking healthcare provider assessment with vaginal $\mathrm{pH}>4.5$ led to correct diagnosis of vaginitis.

Conclusions: The vaginal $\mathrm{pH}$ device can be used as an OTC diagnostic tool by consumers when a vaginal infection is suspected. Vaginal $\mathrm{pH}$ readings would direct patients whether to purchase an antifungal medication or seek professional diagnosis from a healthcare provider. Understanding and use of this vaginal $\mathrm{pH}$ device could reduce inappropriate use of OTC antifungal medications by approximately $50 \%$ and improve the correct diagnosis of vaginitis.

I. Ferris DG, Nyirjesy P, Sobel JD, Soper D, Pavletic A, Litaker MS. Over-the-counter antifungal drug misuse associated with patient-diagnosed vulvovaginal candidiasis. Obstet Gynecol 2002;99:419-25

2. French JL, McGregor JA. Bacterial vaginosis: history, epidemiology, microbiology, sequelae, diagnosis, and treatment. In: Borchardt KA, Noble MA (eds). Sexually Transmitted Diseases: Epidemiology, Pathology, Diagnosis, and Treatment. Boco Raton CRC Press, 1997:4-39 


\title{
The role of an over-the-counter vaginal $\mathrm{pH}$ self-test device package insert: can subjects learn what the device is for and how to use it?
}

\author{
S. Roy, J.C. Caillouette, J.S. Faden and T. Roy
}

Objective: To determine whether subjects could read and understand the package insert of a vaginal pH self-test device to improve self-diagnostic accuracy. 1,2

Methods: This study was performed at eight clinic locations with 206 women of varying ages, ethnicity and education. A package insert was used which explained the indications and clinical facts associated with the use of a vaginal $\mathrm{pH}$ self-test device. A questionnaire was administered to assess comprehension.

Results: This study confirmed that individuals from varied clinics, ages, and ethnic and educational groups could read and understand the package insert. Except for question 14, each of 16 questions had $\geqslant 86 \%$ correct answers. Question 14 indicated that $64 \%$ of women rightly answered that more than $30 \%$ of vaginal itching was due to bacterial infection.

Conclusions: By reading the package insert, subjects correctly understood the role of vaginal $\mathrm{pH}$ as an aid in the diagnosis of vaginal symptoms. This suggests that such tools can improve personal decisions whether to use an over-the-counter antifungal medication or to see a healthcare provider.

I. Ferris DG, Nyirjesy P, Sobel JD, Soper D, Pavletic A, Litaker MS. Over-the-counter antifungal drug misuse associated with patient-diagnosed vulvovaginal candidiasis. Obstet Gynecol 2002;99:4I9-25

2. Ferris DG, Sobel JD, Soper D. Over-the-counter antifungal drug misuse associated with patient-diagnosed vulvovaginal candidiasis. Obstet Gynecol 2002;100:38I-2 


\title{
Scientific poster session. Poster \#9
}

\section{The coexistence of desquamative inflammatory vaginitis and endometrial adenocarcinoma: a case report}

\author{
M.H. Yudin and K.M. Tessler \\ Department of Obstetrics and Gynecology, St. Michael's Hospital, University of Toronto, Canada
}

\begin{abstract}
Objective: To describe the clinical case of a woman with desquamative inflammatory vaginitis and coexisting endometrial adenocarcinoma.

Study design: This is a case study of one patient.

Results: The subject was a 76-year-old Caucasian $\mathrm{G}_{2} \mathrm{P}_{2}$ woman who underwent the menopause at the age of 54 years. She presented to her gynecologist with a 4-month history of profuse, watery vaginal discharge. There was no history of irritative symptoms or postmenopausal bleeding. Physical examination revealed atrophy and the presence of a yellow, watery vaginal discharge. Initial vaginal cultures were negative for bacterial vaginosis, trichomonas vaginalis and yeast. A pelvic ultrasound was performed which showed an endometrial lining of $7 \mathrm{~mm}$, with fluid in the cavity. After persistence of the symptoms, the woman was re-evaluated. Normal saline microscopy demonstrated a paucity of lactobacilli and large numbers of polymorphonuclear leukocytes and parabasal cells, consistent with desquamative inflammatory vaginitis. An endometrial biopsy was performed, and revealed a high-grade endometrial adenocarcinoma. The woman underwent surgical management, and the final pathology was reported as a stage Ic serous endometrial adenocarcinoma, FIGO grade III, with invasion of the outer half of the myometrium.

Conclusions: Desquamative inflammatory vaginitis is an uncommon clinical syndrome of unknown etiology, typically characterized by a long duration of profuse purulent vaginal discharge and exudative vaginitis. It has been reported in pre- and postmenopausal women, and it has been suggested that it may be associated with estrogen deficiency. We were the first group to report this entity in a woman with endometrial cancer. The diagnosis of endometrial adenocarcinoma should be considered in postmenopausal women with desquamative inflammatory vaginitis.
\end{abstract}




\title{
Scientific poster session. Poster \# I0
}

\section{Vaginal candida parapsilosis: pathogen or bystander?}

\author{
P. Nyirjesy, A.B. Alexander and M.V. Weitz \\ Thomas Jefferson University, Philadelphia, USA
}

Objective: Candida parapsilosis is an infrequent isolate on vaginal cultures; its role as a vaginal pathogen remains unstudied. This retrospective study of women with positive cultures for $C$. parapsilosis sought to characterize the significance of this finding and the response to antifungal therapy.

Methods: From a population of women with chronic vulvovaginal symptoms, all individuals with positive fungal isolates from February 200 I to August 2002 were identified. Charts of women with C. parapsilosis cultures were reviewed with regard to subject demographics, clinical presentation and therapeutic response. Mycological cure, defined as a negative fungal culture at the next office visit, and clinical cure, i.e. symptom resolution, were determined for each subject.

Results: A total of 582 women had positive vaginal cultures for 635 isolates; 609 organisms were grown on pure culture. Isolates consisted of $457(72.0 \%)$ C. albicans, 74 (II.7\%) C. glabrata, 54 (8.5\%) C. parapsilosis and 50 (7.9\%) other isolates. The charts of $5 \mathrm{I}$ subjects with $C$. parapsilosis were available for review. Follow-up cultures and clinical information were available in $39(74.5 \%)$ cases.

The median age was 46 years (range 19-86 years); 49/5I (96\%) women were Caucasian; 18/5I (35.3\%) were nulliparous; $21 / 5 \mathrm{I}$ (4I.2\%) were menopausal and of these 19 (90.5\%) were receiving estrogen therapy; 37 (72.5\%) had associated vulvovaginal conditions. Complaints comprised itching in $27 / 5 \mathrm{I}$ (53\%), burning in 22/5 I (43.1\%), abnormal discharge in $1 \mathrm{I} / 5 \mathrm{I}(21.6 \%)$ and dyspareunia in $16 / 5 \mathrm{I}(31.4 \%)$. Microscopy was positive in 9 (17.6\%) cases.

Antifungal treatment resulted in mycological cure for 17/19 women using fluconazole, 7/7 using butoconazole, $6 / 6$ using boric acid and I/I using miconazole. Mycological cure also occurred spontaneously in 6/7 cases (83\%); $24 / 37$ (64.9\%) women with a mycological cure experienced clinical cure.

Conclusions: $C$. parapsilosis, which is often a cause of vaginal symptoms, was observed to respond to a variety of antifungal agents and may even be cleared spontaneously. 


\title{
Prevalence of group B streptococcus colonization and potential for transmission by casual contact in healthy young men and women
}

\author{
S.D. Manning, K. Neighbors, P. Tallman, B. Gillespie, C.F. Marrs, S.M. Borchardt, \\ C.J. Baker, M.D. Pearlman and B. Foxman \\ University of Michigan, Ann Arbor, MI, USA
}

Objectives: Group B streptococcus (GBS) causes disease in the newborn, pregnant women and non-pregnant adults with underlying conditions; as many as $30 \%$ of individuals are asymptomatically colonized. Transmission modes include the vertical, sexual and fecal-oral routes. We describe the prevalence of GBS among college students and co-colonization rates among college roommates. Risk factors associated with site-specific colonization also are highlighted.

Study design: We invited 738 students living in a single dormitory to participate. Students provided urine, throat, vaginal and anal orifice specimens, and completed a questionnaire. Logistic regression identified predictors of GBS colonization. Pulsed-field gel electrophoresis was used to establish the genetic identity of GBS strains.

Results: In all, 462/738 students participated (64\%); 34\% (82/24I) of women and 20\% (43/2II) of men were colonized with GBS in at least one site. Women (OR: 1.9, 95\% Cl: I.2I-2.89), and participants who had ever engaged in sexual activity (OR: $2.5,95 \% \mathrm{Cl}$ : I.39-4.46) were twice as likely to carry GBS. Among the 142 roommate pairs, GBS prevalence at any site was $25.3 \%$ (72/284). If one room mate was positive, the probability that the second was positive was 10/72 (13.9\%).

Conclusion: GBS is prevalent among otherwise healthy college students, and colonization is strongly associated with intimate contact. Direct contact that occurs during cohabitation does not appear to be important for GBS colonization. By contrast, in our previous study of heterosexual college couples in which one sex partner was colonized with GBS, almost half (47.5\%) of their partners also were colonized. 


\title{
Serum proteomic analysis in women experiencing preterm premature rupture of membranes with and without chorioamnionitis
}

\author{
A.P. Murtha ${ }^{1}$, B. Yonish ${ }^{1}$, G.K. Swamy ${ }^{1}$, T.S. Smith ${ }^{1}$, P.V. Basta ${ }^{2}$, C.C. Whisnant ${ }^{2}$, \\ J.L. Bundy ${ }^{2}$, J.L. Stephenson $\mathrm{Jr}^{2}$, D.L. Talley ${ }^{2}$ and R.P. Heine ${ }^{1}$ \\ ${ }^{1}$ Duke University Medical Center, Durham, NC, USA and ${ }^{2}$ RTI International, RTP, NC, USA
}

Objective: Preterm premature rupture of membranes (PPROM) remains a leading cause of neonatal morbidity and mortality. Suspected intra-amniotic infection is a common indication for delivery in PPROM patients. Proteomics offers the ability to examine molecular and functional events. The objective of this study was to determine if differences exist in serum proteins in PPROM patients with and without histologic chorioamnionitis. Methods: All women admitted to hospital with PPROM were eligible for enrollment in this cohort study. Maternal serum was collected daily and at delivery. Placental pathology for evidence of chorioamnionitis was determined by a staff pathologist. Maternal clinical and demographic information was collected prospectively and confirmed by chart review. Of the 96 subjects, a convenience sample of 8 women was selected for analysis: 4 with and 4 without moderate to severe chorioamnionitis. Serum from these subjects was processed to reduce levels of albumin and immunoglobulins, and 2-D gel electrophoresis was performed. Gels were analyzed using computerized densitometry for differential protein expression. Mass spectrometry for protein identification is in process.

Results: Computerized densitometry indicated 8 areas with significantly different intensities in PPROM patients with chorioamnionitis as compared with those without chorioamnionitis.

Conclusion: Proteomic evaluation of maternal serum in women with PPROM may provide a useful way to identify potential areas for research. Further investigation may lead to an improved understanding of the pathogenesis of chorioamnionitis in PPROM cases. 


\title{
Scientific poster session. Poster \#13
}

\section{Risk recognition in a minority population with sexually transmitted disease}

\author{
J.M. Piper, R.N. Shain, S.T. Perdue, J.E. Korte, J.D. Champion and A.E.C. Holden \\ Department of Obstetrics and Gynecology, UTHSCSA, San Antonio, Texas, USA
}

Objectives: Recognition of risk and implementation of beneficial lifestyle changes are key steps in reducing recurrent sexually transmitted diseases (STDs). We assessed current beliefs, risk recognition and willingness to alter behavior in minority women with STDs.

Study design: A total of 820 black and Hispanic women with an active STD (gonorrhea, chlamydia, syphilis or trichomonas) enrolled in an interventional study to reduce STD recurrence. At entry (before intervention), each woman was questioned extensively about interfacing with the healthcare system, previous and potential future reactions to STD infection, further implications of STD infections, and willingness to make behavioral changes. Results: Many women sought health care irregularly for both general health (49\%) and STD testing (59\%); 49\% had no healthcare coverage. Common barriers to health care included cost $(36 \%)$; transportation (26\%); time required ( $28 \%$ too busy, $23 \%$ would not wait long enough); dislike of going (22\%); and belief that symptoms would resolve without care (37\%). Anger at themselves for getting an STD was reported by $88 \%$; $70 \%$ felt stupid; $67 \%$ felt ashamed; $53 \%$ did not know if their current STD could be cured; $94 \%$ worried about the impact of further STDs on their health; and $98 \%$ planned behavioral changes to decrease STD risk (increased condom use $79 \%$, monogamy $47 \%$, more STD testing $38 \%$, abstinence 13\%), although $66 \%$ thought that without any changes they would have little or no chance of getting another STD. However, $66 \%$ worried about catching HIV and $54 \%$ thought this was the most important reason to change behaviour; $92 \%$ reported little or no chance that they had ever been exposed to HIV (based on perceived partner selectivity).

Conclusions: This group of minority women with STDs was concerned about implications of future infections despite their limited knowledge base. They wanted to avoid future risk but had a poor perception of their current risk status. They desired quality health care but reported major barriers to its acquisition. 


\title{
The effect of high group B streptococcus la native antibody on decreased genital colonization
}

\author{
M.A. Krohn, L.A. Meyn, P. Ferrieri, C.J. Baker and S.L. Hillier \\ Baylor College of Medicine, University of Pittsburgh, MN, USA
}

\begin{abstract}
Objectives: We aimed to determine whether high (above the 90th percentile) native antibody to group B streptococcus (GBS) serotype reduced density or cleared genital colonization of that serotype. A previous study showed that high GBS type III antibody prevented recolonization by type III.

Study design: During 1997-200I, 710 women attended four times over I year, in a prospective, observational study conducted at the University of Pittsburgh. High native antibody was above the 90th percentile, and decreased colonization was noted when the semiquantitative density decreased from 3-4+ to I-2+ or broth; clearing colonization was defined as colonization decreasing to negative for the last two visits. Type-specific serum antibody was measured by ELISA at the reference laboratory in Houston. $\mathrm{R} \times \mathrm{C}$ categorical tables were hypothesis-tested using Fisher's exact test. Multivariable modeling was performed with multinomial logistic regression.

Results: At the baseline visit, 9\% of the women had high (54/595) type la antibody and I2\% (74/595) had serotype la colonization at either the vaginal or the rectal site. High native antibody for type la was more likely among women of African-American ethnicity and those who had 2+ children. In addition to these, type la colonization was associated with douching and bacterial vaginosis (BV). After adjusting for ethnicity, douching and $\mathrm{BV}$, the likelihood of having reduced density of type la genital colonization was $70 \%$ higher among women who had high native antibody than among those without elevated antibody $(95 \% \mathrm{Cl}: 0.8-3.1)$. The odds of having la colonization entirely cleared was 2.8 times higher among those with elevated la antibody compared with those without it (95\% Cl: I.2-6.I).
\end{abstract}

Conclusions: These findings suggest that high levels of native antibody to type la GBS is associated with clearing colonization, and are consistent with the Haemophilus influenzae antibody experience. 


\title{
Scientific poster session. Poster \#I5
}

\section{Postmenopausal endometrial tuberculosis: a case report}

\author{
J. Lamb, L. Eckert and H. Deubner \\ Department of Obstetrics and Gynecology, University of WashingtonHarborview Medical Center, Deptartment \\ of Pathology
}

Objectives: Tuberculosis of the upper genital tract is a rare disease in the USA. However, it is a frequent cause of chronic PID and infertility in other parts of the world. A small minority with gynecologic tuberculosis are postmenopausal.' We describe a case of postmenopausal endometrial tuberculosis diagnosed in a 78-year-old woman who presented with vaginal bleeding, and discuss considerations of genital tract tuberculosis.

Study design: This is a case report with presentation of pathologic findings, and a review of pertinent literature. Results: A 78-year-old woman, gravida I and para 0, presented for evaluation of chronic vaginitis which had failed antifungal and estrogen therapy, and with 4 days of postmenopausal bleeding. She had no history of tuberculosis exposure or any upper respiratory symptoms. Physical examination revealed bilaterally painfully inflamed and edematous labia. Areas of atrophy with desquamation at the vaginal introitus and posterior fourchette were noted. The uterus was small and mobile, and no pelvic masses were appreciated. Because biopsy in the office was unsuccessful, and subsequent vaginal ultrasound revealed a thickened endometrial stripe of $12 \mathrm{~mm}$, dilation and curettage were performed. Histology of the endometrial curettings revealed granulomatous endometritis, and acid-fast bacilli were identified. Culture confirmed Mycobacterium tuberculosis. A chest radiograph indicated equivocal potential for granulomatous scarring. The woman received multidrug therapy for 12 months, with complete resolution of symptoms.

Conclusions: Although uncommon in the USA, pelvic tuberculosis should be considered in the differential diagnosis of postmenopausal bleeding, even in a woman without known previous pulmonary infection. Uterine bleeding and vaginitis are characteristic but non-specific manifestations of the infection. Thus, endometrial sampling for histology and microbiologic culture may be required for diagnosis of this treatable condition. This woman responded well to conservative medical management, but it remains controversial whether to recommend combined medical and surgical therapy or medical management alone. ${ }^{2}$

I. Sutherland A.M. Postmenopausal tuberculosis of the female genital Tract. Obstet Gynecol 1982;59:54S

2. Toub DB, Goff BA, Muntz HG. Tuberculous endometritis presenting as postmenopausal bleeding. a case report. J Reprod Med 1991;36:616-8 


\title{
Emerging infection(s) and pregnancy: lethal hemorrhagic fever during an arenavirus outbreak in California
}

\author{
J.A. McGregor and M. Linzey. \\ Cedars-Sinai Medical Center/UCLA, Los Angeles, CA, USA \\ Obstetrix, Tucson, AZ, USA \\ Orange Hospital, Orange, CA, USA
}

Background: Emerging infections (Els) are increasingly recognized as such, for example SARS, hantavirus and other agents recognized in non-endemic areas. Immune adaptations of pregnancy may predispose to uncommon infections that may be difficult to diagnose or may be mistaken for obstetric processes, and may behave more virulently than expected.

Objective: We aimed to increase awareness and promote discussion of Els in pregnancy.

Study design: We report a case of rapidly lethal hemorrhagic fever in a 32-year-old, gravida I, Hispanic woman at 32 weeks' gestation. The woman suffered the acute onset of a 'flu-like illness (FLI) with fever $\left(40^{\circ} \mathrm{C}\right)$, dyspnea, cough, dizziness and myalgia. She was admitted to hospital for "atypical pneumonitis" and received azithromycin. Laboratory findings included neutropenia, thrombocytopenia, elevated hematocrit/haemoglobin, and massively elevated liver enzymes, CPK, BUN and creatinine. She developed progressive petechiae, purpura, multiorgan failure (MOF) with anuria, epistaxis, hematuria, melena and spontaneous bleeding from gums and skin puncture sites. Fetal demise was followed by maternal death despite treatment for septic shock and systemic inflammatory response syndrome. Necropsy showed inflammation and necrosis of placenta, liver and many other organs. This obstetric death occurred contemporaneously with a zoonoic outbreak of Arroyo Blanca/White Bear Canyon arenavirus'.

Conclusions: Els, including hemorrhagic fevers caused by zoonoic arenaviruses, lentiviruses, filoviruses and many other microorganisms, may occur and be comparatively more lethal during pregnancy. Possible El should be considered when caring for pregnant women with otherwise obscure FLI or progressive processes (such as MOF or coagulopathy) complicating pregnancy. 


\title{
Scientific poster session. Poster \# I 7
}

\section{Survey and analysis of membrane stripping practices in presence or absence of group B streptococcus}

\author{
M.A. Pallino and J.A. McGregor \\ Cedars-Sinai Medical Center/UCLA, Los Angeles, CA, USA \\ Obstetrix, Tucson, AZ, USA
}

Background: Membrane stripping at term appears common. Group B streptococcus (GBS) is a perinatal pathogen that is increasingly recognized as causing ascending infection before birth.

Objectives: We aimed to assess the opinions and practices of "expert" obstetricians regarding membrane stripping and GBS carriage in a large community hospital.

Study design: Obstetrical care providers at a large community hospital answered a six-item questionnaire on their opinions and practices after viewing a 3-min ultrasound videotape projection of a cervical examination performed with contract media at term.

Results: Responses were received from 38 obstetricians. Fully 87\% (34/38) stated they had routinely stripped newborns versus II\% who had never done so. Stripping was thought to be an effective means of inducing labor by $45 \%$, versus $39 \%$ who were unsure. Only $13 \%$ would strip membranes in the presence of GBS, versus $79 \%$ who would not; $62 \%$ thought that stripping could introduce pathogens into the uterus, versus $27 \%$ who were unsure. In all, $51 \%$ stated they required an RCT to dissuade them from stripping, versus 13\% who stated a case series would be convincing; $16 \%$ replied that they had observed complications of membrane stripping, such as chorioamnionitis (3), membrane rupture (2), bleeding (3) and perinatal death (I).

Conclusions: Membrane stripping to induce labor at term continues to be commonly performed. In this sample of "expert" practitioners, the majority would not strip membranes in the presence of GBS, believe that pathogens could be introduced into the uterus, and affirm the need for an RCT to dissuade clinicians from membrane stripping. 


\title{
Scientific poster session. Poster \# I 8
}

\section{Group B streptococcus as a cause of near lethal septic shock and pyomyositis treated with protein $\mathrm{C}$ inhibitor}

\author{
J.A. McGregor, A. Madden and D. Platt \\ Cedars-Sinai Medical Center/UCLA, Los Angeles, CA, USA \\ Obstetrix, Tucson, AZ, USA
}

Background: Group B streptococcus (GBS) is a common perinatal pathogen, but it is a rare cause of systemic inflammatory reaction syndrome (SIRS), multiorgan failure (MOF) including acute respiratory distress syndrome (ARDS), and pelvic muscle pyomyositis.

Objectives: We aimed to describe a case of near lethal septic shock with MOF, DIC and multifocal pyomyositis, which required treatment with recombinant protein $C$ inhibitor.

Study design: An 18-year-old Caucasian woman, gravida I, had a surgically uncomplicated I6-week pregnancy termination completed by dilation and extraction. Postoperatively she received $100 \mathrm{mg}$ docycycline twice a day for 7 days, after which she was seen for $38^{\circ} \mathrm{C}$ of fever, dyspnea and chest pain. Chest radiograph showed bilateral effusions and consolidation. Pelvic examination revealed a non-tender uterus with profuse "tomato soup" purulent discharge. Blood cultures (4/4) were positive for GBS. Multiorgan failure with ARDS, renal and liver failure, and elevated CPK followed (APACHE score 9/10). Imaging studies showed no retained products of conception, but multifocal pelvic muscle pyomyositis and abscesses. Treatment included multiple organ support, antimicrobials and recombinant protein $\mathrm{C}$ inhibitor, which was associated with clinical response. Blood culture isolates were reconfirmed as GBS using serologic, microbiologic and molecular techniques, and are being evaluated for atypical virulence factors.

Conclusions: We present an exceptionally severe instance of GBS sepsis with SIRS and pyomyositis, that clinically responded to recombinant protein C inhibitor. GBS may cause extreme maternal morbidity. 


\title{
Scientific poster session. Poster \# I9
}

\section{Perinatal facial palsy: painful, prolonged and often disabling}

\author{
J.A. McGregor and R. Targon \\ Cedars-Sinai Medical Center/UCLA, Los Angeles, CA, USA \\ Obstetrix, Tucson, AZ, USA \\ National Center for Facial Paralysis, Gaithersburg, MD, USA
}

Background: Bell's palsy and Ramsey-Hunt syndrome occur more commonly in pregnancy and the puerperium. Objectives: We aimed to interrogate a uniquely large, national dataset to better characterize how pregnancy affects presentation, duration, care and outcomes of women suffering acute facial palsy. These syndromes are now causally linked to HSV-I and VZV reactivation.

Study design: We investigated a blinded dataset from the National Center for Facial Paralysis (Gaithersburg, MD) from 1998 to 200I. This retrospective analysis was limited to women who had completed detailed assessment and follow-up. We retrospectively analyzed onset timing, indicators of severity, and duration as indicators of comparative morbidity and severity, with historical controls.

Results: Of 1553 patients followed, 782 (50\%) were women of whom $63 \%$ were of reproductive age. Paralysis occurred in the last trimester of pregnancy and in the early puerperium (32\%); $27 \%$ suffered onset in the 9th month of pregnancy. Pregnant women more frequently suffered bilateral facial nerve involvement. In this referred population, no women received treatment with either antiviral or steroid medications. Survival analysis versus historical controls showed that pregnancy-related onset of facial paralysis was significantly slower to resolve and often associated with prolonged and extensive rehabilitation.

Conclusions: In this large dataset, facial paralysis in pregnancy was limited to late pregnancy and the early puerperium, was clinically more severe (bilateral) and less likely to spontaneously resolve, and sufferers did not receive standard treatments commonly administered to non-pregnant individuals. These observations should prompt further investigation to elucidate the pathobiology of these HSV-I and VZV-associated conditions, and to improve treatment of pregnancy-associated facial palsy. 


\title{
Thinking big: the number needed to treat to prevent preterm birth and low birth weight caused by genitourinary tract infection in Los Angeles County, 2003
}

\author{
J.I. French, C. Hobel, M. Henson and J.A. McGregor \\ Cedars-Sinai Medical Center/UCLA, Los Angeles, USA \\ Obstetrix, Tucson, AZ, USA
}

Background: Policymakers, payers, providers and consumers of reproductive care require updated and validated predictive information in order to inform public and private decisions related to care in pregnancy.

Objectives: We aimed to review and analyze current epidemiologic and clinical data to quantify potential approaches for prevention of preterm birth (PTB, short gestation $<38$ weeks), and low birth weight (LBW, $<2500 \mathrm{~g}$ ) in Los Angeles County (LAC).

Study design: Number needed to treat (NNT) models were constructed using epidemiologic data obtained from current or most recent LAC Health Department sources regarding pregnancy numbers, outcomes (PTB, LBW, deaths) and prevalence of candidate conditions in pregnancy or reproductive age populations. NNT for substance abuse was calculated as a comparator.

Results: For asymptomatic bacteruria (ASB, prevalence 5.7\%) NNT to prevent one fetal death was $3 \mathrm{I}$ ( $3 \mathrm{I}$ cases averted); developmental delay 45 ( 2 I cases averted). Table I assumes that all pregnancies leading to births were screened.

Table I

\begin{tabular}{lcccccc}
\hline & ASB & BV & CT & GC & Infection & Substance abuse \\
\hline Prevalence (\%) & 5.7 & 17.5 & 5.8 & 0.8 & 11.4 \\
NNT & 21 & 10 & 11 & 3 & 6 \\
Averted & 368 & 2,611 & 778 & 412 & 4187 & 2590 \\
\hline
\end{tabular}

ASB, asymptomatic bacteruria; BV, bacterial vaginosis; CT, chlamydia; GC, gonorrhea, NNT, number needed to treat

Conclusions: Use of updated findings in LAC informs public health workers, payers, policymakers and consumers in their choices regarding current calculations of (NNT) and numbers needed to screen in order to identify preventable causes of PTB/LBW and to optimize pregnancy outcomes. These calculations must be continuously updated and refined. 


\title{
Scientific poster session. Poster \#2 I
}

\section{Quantitative vaginal fluid sialidase correlates with Nugent's score vaginal Gram stain testing in symptomatic women}

\author{
J.A. McGregor, J.I. French, C. Hastings and S. Johnson \\ Cedars-Sinai Medical Center/UCLA, Los Angeles, CA, USA Obstetrix, Tucson, AZ, and Birmingham, \\ $A L, U S A$
}

Background: Lower reproductive tract infections (LTRIs) and abnormal vaginal microflora are potentially preventable causes of morbid and costly adverse upper reproductive tract infections (URTIs).

Objective: We aimed to compare easy-to-perform, "point of care" measurement of vaginal fluid sialidase to vaginal Gram stain and other standardized clinical testing procedures in symptomatic women at risk for URTI. Study design: Women with symptoms suggesting LRTI were evaluated at three study sites (Denver, CO, Atlanta, GA and Los Angeles, CA). After informed consent, the women were evaluated for vaginal fluid sialidase activity using a well-characterized colormetric test (BV Blue ${ }^{T M}$, Gryphus Diagnostics, Birmingham, AL, USA). Multiple parameters were measured including Nugent's Gram stain score ("gold standard"), vaginal pH, amines, and microscopic examination for "clue cells," yeast forms and trichomonads, as well as Amsel's criteria.

Results: Results from 105 women were analyzed using sialidase activity breakpoint $\geqslant 7.8 \mu$ (positive). Sialidasepositive status correlated with Nugent's score $\geqslant 792 \%$ positive as well as with vaginal $\mathrm{pH}>4.5$ ( $88 \%$ positive), amine positive (79\% positive), milky-white discharge (88\%) and the presence of clue cells (88\%). No sialidase was positive in women with vaginal yeast forms and no woman had trichomoniasis.

Conclusions: In assessment of women with symptoms related to LRTI and to abnormal microflora, we observed that "point of care" testing for sialidase activity $(\geqslant 7.8 \mu)$ was easy to perform and approximated Nugent's score $\geqslant 7$ for clinical diagnosis of bacterial vaginosis and abnormal microflora. We also observed that, similar to Gram stain, sialidase determination appeared more accurate than traditional clinical or microscopic evaluations. 


\title{
Clindamycin in contemporary obstetrics and gynecology: considerations of antibiotic resistance among $\beta$-hemolytic streptococci and anaerobes
}

\author{
K.E. Aldridge ${ }^{1}$, S. Broyles ${ }^{1}$ and R. Master $^{2}$ \\ ${ }^{1}$ LSU Health Sciences Center, New Orleans, LA, USA \\ ${ }^{2}$ Focus Technologies, Herndon, VI, USA
}

Objectives: Clindamycin (Clin) with gentamicin has been used as standard therapy for aerobic-anaerobic infections in obstetrics and gynecology (Ob-Gyn) for decades, but Clin activity against streptococci and anaerobes has changed. This study examines the comparative in vitro activity of Clin against Ob-Gyn isolates of $\beta$-hemolytic streptococci and anaerobes.

Study design: Nationwide susceptibility $(S)$ data to Clin and other pertinent antimicrobials was collated for the years 2000-2002 for $\beta$-hemolytic streptococci (groups B, C, F and G), and over 10 years for anaerobic isolates (\#299) of the B. fragilis group, Prevotella sp., and Peptostreptococcus sp.

Results: Clin-S for group B streptococci during 2000-2002 decreased from 87\% to 81\%; erythromycin-S from $83 \%$ to $70 \%$; and azithromycin-S from $80 \%$ to $66 \%$. For group F streptococci, Clin-S decreased from $95 \%$ to $91 \%$; and erythromycin-S was $90 \%$. For group G streptococci, Clin-S decreased by $3 \%$ and erythromycin-S increased by $5 \%$. Group $C$ streptococcal isolates were the most $S$ to Clin (100\%) and erythromycin (92\%). All streptococcal isolates were $S$ to ampicillin, ceftriaxone (group B), and $>95 \%$ to levofloxacin. Among anaerobes (mostly B. fragilis group) Clin-resistance (Clin-R) was I8\%. Clin-R (with and without coexisting cefoxitin- $R$ ) increased $R$ to ceftizoxime ( $22 \%$ and $12 \%, p=0.02$, respectively); cefotetan ( $56 \%$ and $53 \%, p<0.006$, respectively); cefotaxime ( $29 \%$ and $27 \%, p=0.02$, respectively); piperacillin ( $32 \%$ and $28 \%, p=0.005$, respectively); and ciprofloxacin ( $19 \%$ and $17 \%, p=0.02$, respectively). Odds ratios for cross-resistance ranged from 3.2 to 14.5. No decreased $S$ was noted for piperacillin-tazobactam nor for meropenem.

Conclusions: These data indicated that for the commonly encountered Ob-Gyn pathogens ( $\beta$-hemolytic streptococci and anaerobes) clindamycin-S continues to decrease. Moreover, clindamycin-R serves as a marker for cross-resistance to $\beta$-lactam agents among anaerobes. These data may give reason for reconsideration of clindamycin as first-line therapy. 


\title{
Scientific poster session. Poster \#23
}

\section{Urinary tract infections after gynecologic surgery}

\author{
C.S. DiMarco, A.J. Croak, D.S. DiMarco, C.J. Klingele, R.A. Lee and J.B. Gebhart \\ Mayo Clinic, Rochester, MN, USA
}

Objective: We aimed to report the occurrence of and organisms responsible for urinary tract infections (UTI) following gynecologic operations.

Study design: Records were reviewed of women having gynecologic operations in the year 2002 to find cultureproven UTI during the first 6 weeks postoperatively. UTI was defined as symptomatic with urine cultures $\geqslant 100000 \mathrm{cfu} / \mathrm{ml}$.

Results: A total of 816 gynecologic operations were undergone by local women, $6 \%$ (50) of whom were diagnosed as having UTI. The most common single causative organism was a coagulase-negative staphylococcus (12,24\%), followed by Escherichia coli (10,20\%). One case of Enterobacter cloace urosepsis was reported, and 9 infections with two infective organisms were cultured, 6/9 (67\%) of which were associated with prolapse surgery. Enterococcus $(5 / 9,51 \%)$ was most commonly associated with dual infection. Surgery for uUrinary incontinence or prolapse or both was performed in $28(56 \%)$ cases; other benign conditions were present in 16 (32\%) cases; and gynecologic cancer was recorded in 6 (12\%) cases. E coli in 6/16 (38\%) was most commonly related to benign surgeries. The mean age was 54 years (range 20-83 years) and 25 (50\%) women were postmenopausal. Surgical approaches included 26 vaginal, 19 abdominal, 2 laparoscopic and 3 minor procedures. Staphylococcal UTI was seen equally between vaginal and abdominal cases. Of these women, 23 (46\%) had preoperative urinalysis and 4 were infected, with only I having a similar organism ( $E$ coli) postoperatively. Interestingly, $32(64 \%)$ required intraoperative urologic instrumentation; 5/32 (16\%) required stent placement; and $15 / 32(47 \%)$ required cystoscopy or suprapubic catheter (SPC) insertion or both (30/32, 94\%). Staphylococcus was most frequently associated with a history of SPC, in $8 / 32(25 \%)$ women.

Conclusions: Postoperative UTI complicated $6 \%$ of all gynecologic surgical operations. Staphylococcus was the most common organism. Vaginal operations, surgery for incontinence or prolapse and use of SPC may be risk factors for postoperative UTI. 


\title{
Non-gonococcal, non-chlamydial cervicitis: an under- appreciated cause of vaginal discharge
}

\author{
L. Chesney Thompson, K. Truelock and R.S. Gibbs
}

Objectives: We aimed to identify distinguishing characteristics of non-gonococcal, non-chlamydial cervicitis (NGNCC), to improve diagnosis and management.

Study design: This was a case series study in which we reviewed 16 charts from our referral practice for vulvovaginal disorders.

Results: All the subjects were premenopausal and privately insured; 4 were single, 12 married, 2 were Hispanic and 14 Caucasian. All the women had symptoms of chronic vaginal discharge with or without burning, itching or dyspareunia. Evaluation revealed mucopurulent discharge in the endocervix, with increased white blood cells (WBCs) on microscopy, and $\mathrm{pH}$ was elevated in 9 of 15 . Wet preparations were negative for bacterial vaginosis (BV), trichomoniasis and yeast infection, but yeast cultures were positive in 4 of 15 . All were negative for Chlamydia trachomatis and Neisseria gonorrhoeae. Cultures revealed group B streptococcus in 2/6 and coagulasenegative staphylococcus species in 4/6. Empiric treatment and responses are shown in Table I.

Cryotherapy was used in 5 cases refractory to antibiotics, with I cure and I partial response.

Table I Antibiotic effectiveness

\begin{tabular}{lccc}
\hline Antibiotic & Number of courses & Number of cures & Number of partial responses \\
\hline Clindamycin & 13 & 2 & 5 \\
Quinolones & 13 & 0 & 3 \\
Metronidazole & 9 & 0 & 0 \\
Azithromycin & 5 & 0 & 2 \\
Doxycycline & 3 & 0 & 0 \\
Penicillins & 2 & 0 & 0 \\
Tetracycline & 1 & 0 & 1 \\
\hline
\end{tabular}

Conclusions: NGNCC is an under-appreciated condition occurring commonly in women entering a referral practice with complaints of vaginal discharge. No current criteria exist for diagnostic classification. Women previously received multiple diagnoses and treatments, and routine bacterial cultures do not appear beneficial initially. We propose that diagnostic criteria include:

- mucopurulent cervical discharge, usually with ectopy

- increased WBCs on endocervical microscopy

- negative testing for chlamydia and gonorrhoeal infection

- no cervical or pelvic tenderness

- exclusion of yeast, BV, trichomonas and desquamative inflammatory vaginitis as etiology for the symptoms.

As NGNCC may present in routine office gynecology, we would like to generate a collective endeavor to increase recognition, standardize diagnostic criteria, and establish consistency in therapeutic approaches to this entity amongst interested centers. 


\title{
Scientific poster session. Poster \#25
}

\section{Genetic diversity of vaginal Lactobacillus spp. determined by pulsed-field gel electrophoresis in women at 20 and 30 weeks' gestation}

\author{
J.E. Warner, R.A. Ross, M.L. Delaney, A.M. DuBois and A.B. Onderdonk \\ Channing Laboratory, Brigham,Women's Hospital, Harvard Medical School, Boston, MA, USA.
}

\begin{abstract}
Objectives: A number of studies have established that the predominant bacterial species within the vagina are Lactobacillus spp. and that these are considered the organisms responsible for maintaining a healthy vaginal environment. Understanding some of the possible roles that Lactobacillus spp. may play in preterm delivery is the key purpose of this research.

Study design: We studied genomic diversity, estimated by pulsed-field gel electrophoresis (PFGE), and how it relates to preterm birth. Genomic DNA of II 3 Lactobacillus sp. isolates from vaginal swabs taken at 20 and 30 weeks' gestation from 4I women were cleaved with Smal restriction endonuclease and resolved in an agarose gel by PFGE. Participants were separated into pregnancy outcome groups defined as term (delivered at $\mathbf{3 7}$ or more weeks' gestation) or preterm (delivered at less than 37 weeks' gestation). These groups were then compared with PFGE types.
\end{abstract}

Results: From II 3 isolates, 103 unique PFGE patterns emerged (91\%) with a diversity index of $D_{H}=99.5$. A $70 \%$ similarity index resulted in 86 PFGE types where the majority of the types $(63 / 86,73 \%)$ consisted predominantly of $\mathrm{I}$ isolate harbored by a single participant. The same clones were sustained over the 10 weeks by $79 \%$ of women evaluated at both 20 and 30 weeks. There appeared to be no relationship between PFGE patterns and preterm delivery.

Conclusion: Most participants appeared to harbor their own clone and sustained these clones over a 10 -week period. There were no PFGE pattern correlates for preterm delivery. 


\title{
Quantitative method for determining presence of bacteria in placenta samples
}

\author{
A. DuBois, M. Delaney and A. Onderdonk
}

Objectives: Intra-uterine infection that contributes to delivery before the end of the second trimester is usually documented by culture of the amniotic fluid or amniotic membranes. If specific organisms initiate the fetal inflammatory response leading to preterm labor, then efforts to identify all of these organisms are necessary. By culturing the chorionic plate rather than swabbing the chorioamnion junction, we hoped to find an initiator of preterm delivery.

Study design: After pulling away intact amnion, a piece of chorion and underlying tissue was cut and placed in a vial for flash freezing and storage at $-80^{\circ} \mathrm{C}$. A portion of the placenta was weighed and homogenized in PBS. Serial dilutions were made and plated onto selective and non-selective media. Plates were incubated in the appropriate atmosphere and colony types were enumerated and selected for identification. If no bacteria were colonized, the remaining portion of placenta tissue was digested and DNA was extracted. The presence of an 800 kb PCR product (using universal bacterial primers) was considered positive.

Results: As part of an ongoing NIH study, 346 placental samples were processed for bacterial identification. Of these, 204 were culture-negative. Bacteria were cultured from the remaining 142 samples, and of these 62 were considered culture-positive in that the bacteria cultured were single known pathogens or known pathogens 2 logs higher than the non-pathogens present. Mycoplasma hominis (Mh) or Ureaplasma urealyticum (Uu) or both were cultured from 28 samples; I I samples were culture-positive for Mh; 8 samples for Uu; I sample for Mh and Uu; 8 for Mh or Uu or both, with other bacteria. For those samples that were culture-negative, PCR assay is under way to confirm the absence of bacteria.

Conclusion: This method of culturing the chorionic plate has the potential to provide the best information about what stimulates the fetal inflammatory response. 


\title{
Scientific poster session. Poster \#27
}

\section{Quantitative and qualitative assessment of vaginal microbiology in women with and without vulvodynia}

\author{
M.L. Delaney, B.L. Harlow and A.B. Onderdonk
}

Objectives: Vulvodynia is a syndrome characterized by unexplained burning, knife-like pain or excruciating pain on contact with the vulvar region that results in severe physical and psychological distress. A diagnosis of vulvodynia is made after excluding other explainable vulvar pain disorders including candidiasis, inflammatory vaginitis, sexually transmitted diseases, dermatoses, menopausal dyspareunia or other pelvic pain syndromes. We present preliminary findings from an ongoing $\mathrm{NIH}$-funded study to determine etiological predictors of this disorder.

Study design: We identified 100 symptomatic cases and 100 age-matched controls from among 4000 women, aged 18-64 years, from the general population. Of these, 31 cases and 13 controls (thus far) have provided vaginal swab samples to quantitatively and qualitatively assess the presence of microorganisms. Vaginal smears from each subject were Gram stained and evaluated using the Nugent criteria to detect bacterial vaginosis (BV). Results: A comparison of vaginal $\mathrm{pH}$, total aerobic and anaerobic bacterial counts, and individual genera comprising the total counts showed no significant differences between the two groups of subjects. Analysis of the frequency of isolation for the various bacterial genera present showed a significantly higher prevalence of group $D$ streptococcus (GDS) in the subjects with vulvodynia when compared with the control subjects $(64 \%$ versus $31 \%, p=0.04$ ). Furthermore, when we compared the frequency of GDS in vulvodynia subjects with previously published data from overtly healthy subjects (expected prevalence $=15-20 \%$ ) the association remained highly significant. In contrast, Prevotella sp. and Actinomyce $s p$. were isolated with greater frequency from control subjects than those with vulvodynia $(p<0.01)$.

Conclusions: Preliminary data suggest that the vaginal microflora of women with vulvodynia may be unique. Furthermore, the association of GDS with vulvodynia appears to far exceed that observed for candidiasis, BV or an elevated $\mathrm{pH}$, all of which have been suggested as risk factors for vulvodynia. 


\title{
Fusobacterium nucleatum induces premature and term stillbirth in pregnant mice: implication of oral bacteria in preterm birth
}

\author{
Y.W. Han ${ }^{1}$, R.W. Redline ${ }^{2}$, M. Li ${ }^{1}$, L. Yin ${ }^{1}$, G.B. Hill ${ }^{4}$ and T.S. McCormick ${ }^{3}$ \\ Departments of ${ }^{1}$ Periodontics, ${ }^{2}$ Pathology and ${ }^{3}$ Dermatology, Case Western Reserve University and \\ ${ }^{4}$ Department of Obstetrics and Gynecology, Duke University Medical Center
}

Background: Fusobacterium nucleatum is a Gram-negative anaerobe associated with periodontal disease (PD). It is also implicated in preterm birth (PTB) and is isolated from amniotic fluid (AF), placenta and chorioamnion. Epidemiological studies have indicated PD as a potential risk factor for PTB. It has been hypothesized that transient bacteremia with oral $F$. nucleatum might infect the fetoplacental unit.

Objectives: We aimed to evaluate the invasiveness of various fusobacteria for human epithelial and endothelial cells and the effects of $F$. nucleatum, injected intravenously, on pregnancy outcomes in mice.

Study design: Respiratory, AF, placental and vaginal isolates of fusobacteria were assayed for invasion of human umbilical vein endothelial and oral mucosal epithelial cells. Respiratory and AF isolates of $F$. nucleatum were injected intravenously into pregnant CF-I mice at day 16-17 of gestation. The effects were monitored by quantitative bacterial cultures of murine tissues, immunohistochemistry and transmission electron microscopy to locate $F$. nucleatum in mouse placentas, and the death rate of mouse pups at delivery.

Results: Respiratory and intrauterine isolates of $F$. nucleatum invaded endothelial and epithelial cells, but $F$. gonidiaformans from the vagina were non-invasive. Intravenously injected $F$. nucleatum proliferated in placental tissues. This infection caused significant levels of preterm fetal death, stillbirths, and early deaths of liveborn pups. In contrast, the organisms were unable to persist in the liver or spleen. In the placenta, $F$. nucleatum spread sequentially from the blood vessels to the decidua basalis, placental membranes and, finally, amniotic fluid.

Conclusions: We fulfilled Koch's postulates to identify $F$. nucleatum as a causative agent of adverse pregnancy outcome. The invasiveness of $F$. nucleatum for human epithelial and endothelial cells may be a virulence factor for placental infection. Our study further supports the hypothesis that $F$. nucleatum may transmit hematogenously from the oral cavity to the placenta and strengthens the link between PD and PTB. 


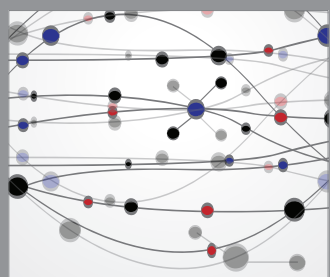

The Scientific World Journal
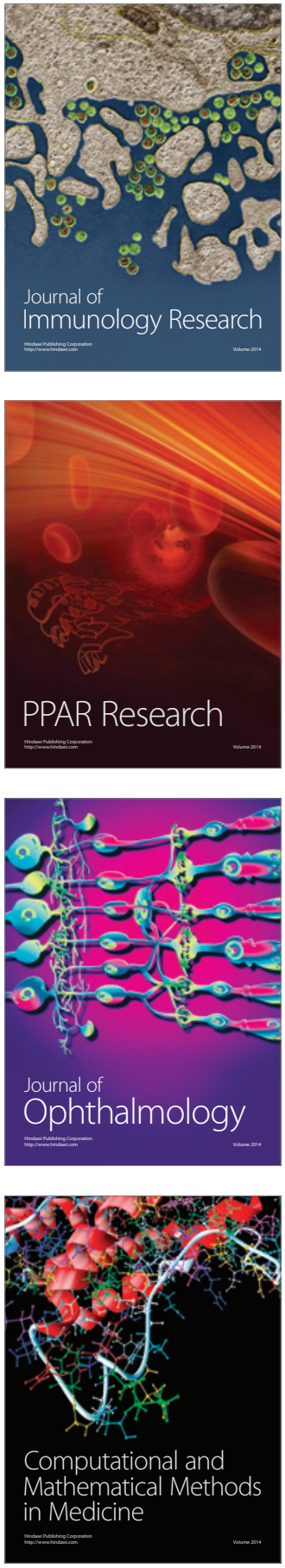

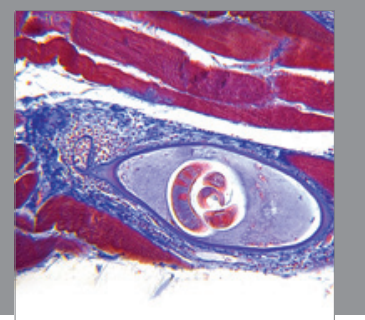

Gastroenterology

Research and Practice
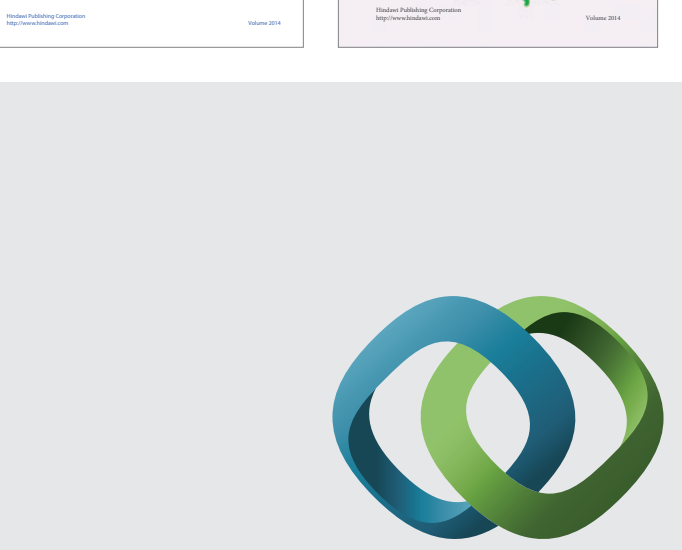

\section{Hindawi}

Submit your manuscripts at

http://www.hindawi.com
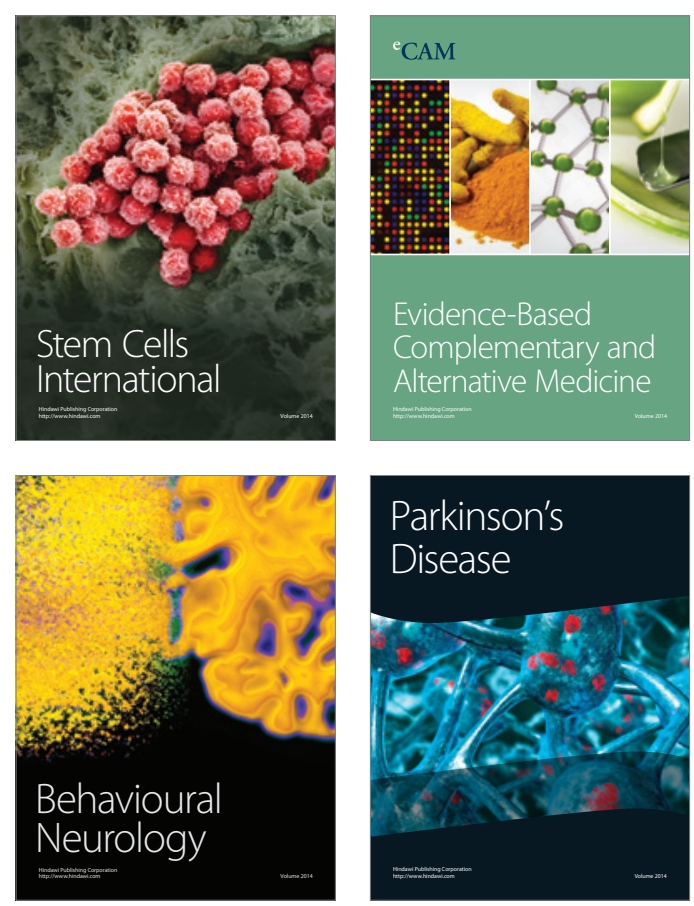

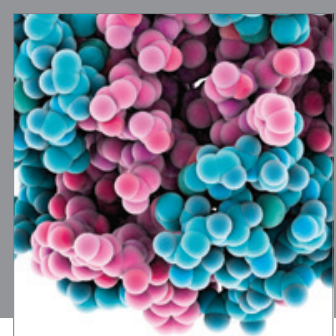

Journal of
Diabetes Research

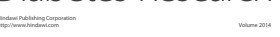

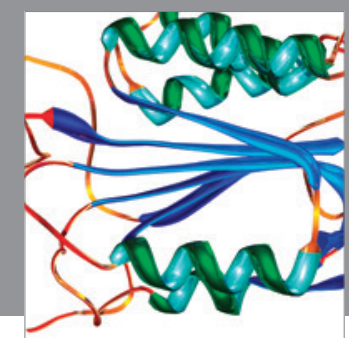

Disease Markers
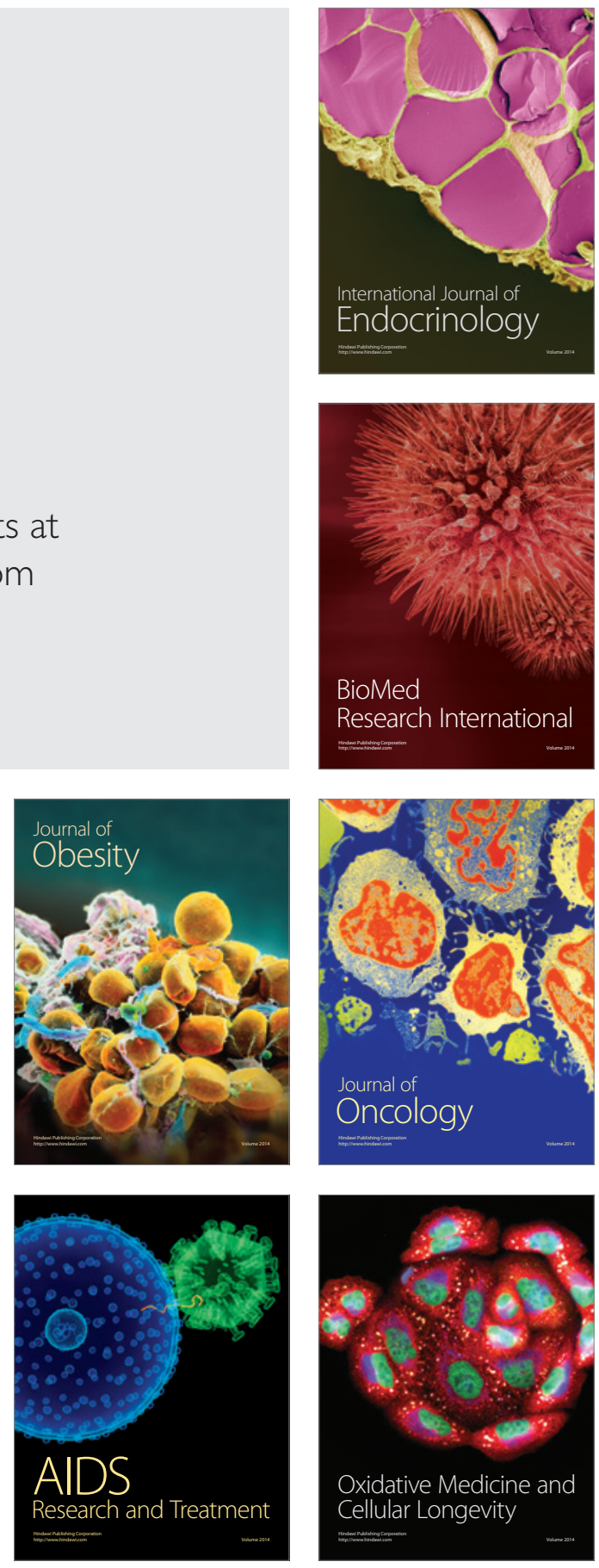